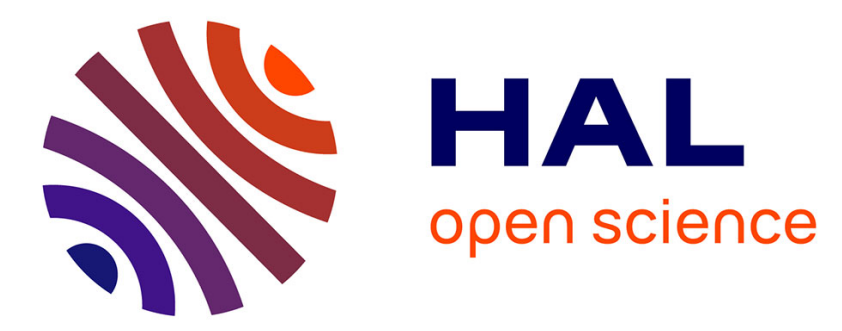

\title{
Fixed-time stabilization of fuzzy neutral-type inertial neural networks with time-varying delay
}

Chaouki Aouiti, Qing Hui, Hediene Jallouli, Emmanuel Moulay

\section{To cite this version:}

Chaouki Aouiti, Qing Hui, Hediene Jallouli, Emmanuel Moulay. Fixed-time stabilization of fuzzy neutral-type inertial neural networks with time-varying delay. Fuzzy Sets and Systems, 2021, 411, pp.48-67. 10.1016/j.fss.2020.10.018 . hal-03179755

\section{HAL Id: hal-03179755 \\ https://hal.science/hal-03179755}

Submitted on 24 Mar 2021

HAL is a multi-disciplinary open access archive for the deposit and dissemination of scientific research documents, whether they are published or not. The documents may come from teaching and research institutions in France or abroad, or from public or private research centers.
L'archive ouverte pluridisciplinaire HAL, est destinée au dépôt et à la diffusion de documents scientifiques de niveau recherche, publiés ou non, émanant des établissements d'enseignement et de recherche français ou étrangers, des laboratoires publics ou privés. 


\title{
Fixed-time stabilization of fuzzy neutral-type inertial neural networks with time-varying delay
}

\author{
Chaouki Aouiti ${ }^{\mathrm{a}}$, Qing Hui ${ }^{\mathrm{b}}$, Hediene Jalloulia ${ }^{\mathrm{a}}$ Emmanuel Moulay ${ }^{\mathrm{c}, \mathrm{b}}$ \\ ${ }^{a}$ University of Carthage, Faculty of Sciences of Bizerta, Department of Mathematics, Research Units of Mathematics and \\ Applications UR13ES47, BP W, 7021 Zarzouna, Bizerta, Tunisia. \\ ${ }^{b}$ Department of Electrical and Computer Engineering, University of Nebraska-Lincoln, Lincoln, NE 68588-0511, USA \\ ${ }^{c}$ XLIM (UMR CNRS 7252), Université de Poitiers, 11 bd Marie et Pierre Curie, 86073 Poitiers Cedex 9, France.
}

\begin{abstract}
This paper addresses the problem of fixed-time stabilization for a class of fuzzy neutral-type inertial neural networks (FNTINNs) with time-varying delay. By using a novel fixed-time stability theorem for dynamical systems, two different feedback control laws are designed to ensure the fixed-time stabilization of FNTINNs with time-varying delay. The proposed theoretical results can lead to a better upper settling-time estimation compared to existing results. Finally, three simulation examples are provided to illustrate the validity of the proposed theoretical results.
\end{abstract}

Keywords: Inertial neural networks, fuzzy neural networks, time-varying delay, fixed-time stability

\section{Introduction}

In 1996, Yang studied fuzzy neural networks (FNNs) in 1, 2] as another type of cellular neural network model which combines fuzzy logic involving the furry rules with neural networks. Recent studies revealed that FNNs are a very useful model for image processing problems which are a cornerstone in pattern recognition [3, 4, 5, 6. These applications heavily depend on the dynamical behaviors of FNNs, and hence, it is of importance to gain the knowledge of their dynamics in local and global scales. It is known that in both biological and artificial neural networks, the delays arise due to information processing latency. The existence of time delays may lead to oscillation, divergence or instability type of dynamical behavior, which could be harmful to the stability of dynamical systems. Therefore, the study of neural dynamics taking into account the delays becomes imperative to construct high-quality NNs. Recently, many analytical results were obtained for FNNs with various axonal signal transmission delays [7, 8, 9. The problem of exponential and almost sure exponential stability for stochastic fuzzy delayed Cohen-Grossberg neural networks was investigated in 7 where the authors establish that fuzzy systems do have more advantages than non-fuzzy systems. In [10, the problem of finite-time synchronization for a class of FNNs with time-varying and proportional delays has been investigated. Note that several previous works mainly study FNNs with only first-order derivative, while it is important to introduce an inertial term to obtain the fuzzy inertial model. In 1997, Wheeler and Schieve were the first one to publish an article [11] about inertial neural networks (INNs), which are more complex than all kinds of NNs. Since then, many results of INNs have been obtained by many researchers, see for instance 12,13 .

The stability problem of dynamical systems can be classified into two categories. The first one is the infinite-time stability such as the asymptotic stability [14] or the exponential stability [15] where the solutions of the dynamical system converge to the equilibrium point in a limit way. The other one is the finite-time stability where the solutions of the dynamical system reach the equilibrium point in finite time [16, 17. The

Email addresses: chaouki.aouiti@fsb.rnu.tn (Chaouki Aouiti), qing.hui@unl.edu (Qing Hui), jalouli.hedienne@fsb.rnu.tn (Hediene Jallouli), emmanuel.moulay@univ-poitiers.fr (Emmanuel Moulay) 
time function which indicates the time for reaching the equilibrium is called the settling-time. The finitetime stability has a great importance in real-world applications of engineering systems such as robotics, optimization problems, pattern recognition, and vehicle systems or spacecraft systems [18, 19, 20, 21]. It is worth noting that finite-time stability of FNTINNs has been investigating in 22 .

It has been recognized that the settling-time estimation in finite-time stabilization problems relates essentially to the initial value of the system. This fact results in disadvantages for real-world applications because it is difficult or even impossible to obtain in advance the initial conditions in many practical systems 23]. To overcome this issue, Polyakov has developed the concept of fixed-time stability in [24] where the settling-time is bounded by a constant and does not depend on the initial conditions. The study of fixed-time stabilization for a general class of NNs was investigated in [25].

To the best of our knowledge, the stability analysis of neutral-type inertial neural networks with delays has been investigated in 26 and the finite-time synchronization for various kinds of INNs has been obtained in [27, 28]. But there is hardly any paper that considered the fixed-time stabilization for FNTINNs with time-varying delay. Inspired by the aforementioned analysis, this paper aims at investigating the fixedtime stabilization of FNTINNs with time-varying delays. By using a novel fixed-time stability theorem of dynamical systems and Lyapunov method, two different control protocols are designed to guarantee the fixed-time stabilization of FNTINNs with time-varying delay. Also, the proposed theoretical results of this article present a more accurate upper settling-time estimation compared to known results [23, 29, 30]. The highlights and the main contributions of this paper are embodied in the following aspects:

- FNTINNs with time-varying delay can be rewritten as first-order differential systems. In the light of the double layer structure of FNTINNs with time-varying delay, two controllers are proposed for the fixed-time stabilization depending on whether the activation functions are bounded or not. The settling-time can be overestimated regardless of the initial conditions of the FNTINNs.

- The fixed-time stabilization of FNTINNs with time-varying delay is considered in this paper. To our best knowledge, there is no result in the literature considering the fixed-time stabilization for a class of FNTINNs with time-varying delay, which is vital in both theories and applications and also a very challenging problem.

- Numerical examples show that the new fixed-time stabilization theorems developed in this paper can provide a more accurate upper settling-time estimation for NNs compared to many known results in the literature.

The rest of this article is organized as follows. After some preliminaries given in Section 2, the model description is presented in Section 3 . Then the main theoretical results are derived in Section 4 . In Section 5 . numerical simulations are provided to verify the theoretical results. Finally, a conclusion is reached in Section 6

\section{Preliminaries}

In the remainder of the paper, we denote $\mathbb{R}^{+}$the set of positive real numbers. Let us recall some results on finite-time stability and fixed-time stability. Consider the following ordinary differential equation

$$
\begin{aligned}
\dot{y}(t) & =f(y(t)), \quad y(t) \in \mathbb{R}^{n} \\
y(0) & =y_{0}
\end{aligned}
$$

where $f$ is a continuous function such that $f(0)=0$.

Definition 1. 17] The system (1] is finite-time stable if there exists $\mathbf{T}>0$ dependent on the initial conditions such that $\lim _{t \rightarrow \mathbf{T}}\|y(t)\|=0$ and $\|y(t)\| \equiv 0$ for all $t>\mathbf{T}$. The function $\mathbf{T}$ is called the settlingtime.

Definition 2. 31] The system (1) is fixed-time stable if: 
(1) it is finite-time stable;

(2) the settling-time function $\mathbf{T}$ is upper bounded by a constant $\mathbf{T}^{\max }>0$, i.e., $\mathbf{T} \leq \mathbf{T}^{\max }$.

Definition 2 means that the settling-time function $\mathbf{T}$ does not depend on the initial conditions. Next, we list several existing results for finite-time stability and fixed-time stability of (1) by using Lyapunov functions.

Lemma 1. [31, Theorem 2.3] If there exists a continuously differentiable, positive-definite, radially unbounded function $\mathbb{V}: \mathbb{R}^{n} \rightarrow \mathbb{R}^{+}$such that

$$
\dot{\mathbb{V}}(y(t)) \leq-\underline{b} \mathbb{V}(y(t))^{\kappa}-\underline{c} \mathbb{V}(y(t))^{\mu}
$$

where $y \in \mathbb{R}^{n}$ and $\underline{b}, \underline{c}>0,0<\kappa<1<\mu$, then the system (1) is fixed-time stable and the settling-time satisfies

$$
\mathbf{T} \leq \mathbf{T}_{1}^{\max }:=\frac{1}{\underline{b}(1-\kappa)}+\frac{1}{\underline{c}(\mu-1)} .
$$

$\mathbb{V}$ is called a Lyapunov function for the system (1).

Lemma 2. [32, Lemma 3] If there exists a continuously differentiable, positive-definite, radially unbounded function $\mathbb{V}: \mathbb{R}^{n} \rightarrow \mathbb{R}^{+}$such that

$$
\dot{\mathbb{V}}(y(t)) \leq-\underline{b} \mathbb{V}(y(t))^{\kappa}-\underline{c} \mathbb{V}(y(t))^{\mu}
$$

where $y \in \mathbb{R}^{n}$ and $\underline{b}, \underline{c}>0,0<\kappa<1<\mu$, then the system (1) is fixed-time stable and the settling-time satisfies

$$
\mathbf{T} \leq \mathbf{T}_{2}^{\max }:=\frac{1}{\underline{b}}\left(\frac{\underline{b}}{\underline{c}}\right)^{\frac{1-\kappa}{\mu-\kappa}}\left(\frac{1}{\underline{b}(1-\kappa)}+\frac{1}{\underline{c}(\mu-1)}\right) .
$$

Lemma 3. [32, Lemma 4] Without loss of generality, if $\kappa=1-\frac{1}{2 \theta}$ and $\mu=1+\frac{1}{2 \theta}$ with $\theta>1$, then the upper bound of the settling-time function $\mathbf{T}_{3}^{\max }$ of the system (1) can be estimated by

$$
\mathbf{T}_{3}^{\max }=\frac{\theta \pi}{\sqrt{\underline{b} \underline{c}}} .
$$

Lemma 4. [32, Theorem 1] If there exists a continuously differentiable, positive-definite, radially unbounded function $\mathbb{V}: \mathbb{R}^{n} \rightarrow \mathbb{R}_{+}$such that

$$
\dot{\mathbb{V}}(z(t)) \leq-\underline{a} \mathbb{V}(z(t))-\underline{b} \mathbb{V}(z(t))^{\kappa}-\underline{c} \mathbb{V}(z(t))^{\mu}
$$

where $y \in \mathbb{R}^{n}$ and $\underline{a}, \underline{b}, \underline{c}>0,0<\kappa<1$ and $\mu>1$, then the system (1) is fixed-time stable and the settling-time satisfies

$$
\mathbf{T} \leq \mathbf{T}_{4}^{\max }:=\frac{1}{\underline{a}(1-\kappa)} \log \left(\frac{\underline{b}+\underline{a}}{\underline{b}}\right)+\frac{1}{\underline{a}(\mu-1)} \log \left(\frac{\underline{c}+\underline{a}}{\underline{c}}\right) .
$$

We conclude this section with a lemma of inequalities that is useful later in the paper.

Lemma 5. 32] If $\xi_{1}, \ldots, \xi_{n} \geq 0,0<\theta_{1}<1$ and $\theta_{2}>1$, the following inequalities hold

$$
\sum_{\ell=1}^{n} \xi_{l}^{\theta_{1}} \geq\left(\sum_{\ell=1}^{n} \xi_{l}\right)^{\theta_{1}} ; \sum_{\ell=1}^{n} \xi_{l}^{\theta_{2}} \geq n^{1-\theta_{2}}\left(\sum_{\ell=1}^{n} \xi_{l}\right)^{\theta_{2}}
$$




\section{Model description}

For $i=1, \cdots, n$, the following FNTINN with time-varying delay is considered:

$$
\begin{aligned}
\frac{d^{2} y_{i}(t)}{d t^{2}}= & -a_{i} \frac{d y_{i}(t)}{d t}-b_{i} y_{i}(t)+\sum_{j=1}^{n} c_{i j} f_{j}\left(y_{j}(t)\right)+\sum_{j=1}^{n} d_{i j} f_{j}\left(y_{j}\left(t-\tau_{j}(t)\right)\right) \\
& +\sum_{j=1}^{n} \eta_{i j} \int_{-\infty}^{t} K(t-s) f_{j}\left(y_{j}(s)\right) d s+\sum_{j=1}^{n} e_{i j} f_{j}\left(\dot{y}_{j}\left(t-\tau_{j}(t)\right)\right) \\
& +\sum_{j=1}^{n} g_{i j}(t) v_{j}(t)+\bigwedge_{j=1}^{n} T_{i j}(t) v_{j}(t)+\bigwedge_{j=1}^{n} \alpha_{i j} f_{j}\left(y_{j}\left(t-\tau_{j}(t)\right)\right) \\
& +\bigvee_{j=1}^{n} \beta_{i j} f_{j}\left(y_{j}\left(t-\tau_{j}(t)\right)\right)+\bigwedge_{j=1}^{n} \gamma_{i j} f_{j}\left(\dot{y}_{j}\left(t-\tau_{j}(t)\right)\right)+\bigvee_{j=1}^{n} \delta_{i j} f_{j}\left(\dot{y}_{j}\left(t-\tau_{j}(t)\right)\right) \\
& +\sum_{j=1}^{n} g_{i j}(t) v_{j}(t)+\bigvee_{j=1}^{n} S_{i j}(t) v_{j}(t)+\bigwedge_{j=1}^{n} T_{i j}(t) v_{j}(t)+I_{i}(t),
\end{aligned}
$$

where the second derivative is called an inertial term of the system $(2) ; y_{i}(t) \in \mathbb{R}$ denotes the state of the $i^{\text {th }}$ neuron at time $t ; a_{i}>0, b_{i}>0$ are constants; $c_{i j}, d_{i j}, e_{i j}$ and $\eta_{i j}$ are connection weights related to the neurons without delay and with delay respectively; $K j($.$) is the delay kernel; g_{i j}$ denotes the feedforward template; $\alpha_{i j}, \beta_{i j}, \gamma_{i j}, \delta_{i j}$ and $T_{i j}, S_{i j}$ are elements of the fuzzy feedback MIN template, fuzzy feedback MAX template, fuzzy feed-forward MIN template and fuzzy feed-forward MAX template, respectively; $\bigwedge$ and $\bigvee$ denote the fuzzy AND and fuzzy OR operation, respectively; $v_{j}$ is the input of the $j^{\text {th }}$ neuron with $j=1, \cdots, n ; f_{j}(t)$ denotes the activation function of $j^{t h}$ neuron at time $t ; \tau_{j}(t)$ is the time-varying delay of $j^{\text {th }}$ neuron at time $t$; and $I_{i}$ is the bias on the $i^{\text {th }}$ neuron.

Remark 3.1. $\bigwedge$ and $\bigvee$ denote the fuzzy $A N D$ and fuzzy $O R$ operations, respectively. A template is a matrix composed of each set of connection parameters in FNNs. The feedback template represent the delay link parameters of the neurons, while the feed-forward template represent the link parameters of the neurons. Therefore, the fuzzy feed-forward MAX template and fuzzy feed-forward MIN template refer to the delay link parameters with the fuzzy $O R$ and fuzzy AND operations, respectively; feedback MAX template and feedback $M I N$ template refer to the link parameters with the fuzzy OR and fuzzy AND operations, respectively. Unlike INNs structures investigated in [33, 34, 35], FNTINN has fuzzy logic between its template and input AND/OR output besides the "sum of product" operation. Meanwhile, many studies have been revealed that FINN is a useful paradigm for image processing problems, which is a cornerstone in image processing and pattern recognition. Therefore, it is of great importance to analyze the dynamical behaviors of FNTINN both in theory and applications [36].

The initial conditions of the system (2) are given by

$$
y_{i}(s)=\check{\varphi}_{i}(s), \quad \frac{d y_{i}(s)}{d t}=\check{\psi}_{i}(s), \quad s \in[-\tau, 0], i=1, \cdots, n
$$

where $\check{\varphi}_{i}($.$) and \check{\psi}_{i}($.$) are real-valued continuous functions on [-\tau, 0]$ with

$$
\tau=\max _{1 \leq j \leq n}\left\{\sup _{t \in \mathbb{R}_{+}} \tau_{j}(t)\right\} .
$$

Now, the following assumptions are introduced to derive the main results of this paper:

$\left(\mathbf{A}_{1}\right)$ The activation functions $f_{j}($.$) with j=1, \cdots, n$ satisfy the following Lipschitz condition: for $\xi, \tilde{\xi} \in \mathbb{R}$, there exists $\ell_{j}>0$ such that

$$
\frac{\left|f_{j}(\xi)-f_{j}(\tilde{\xi})\right|}{|\xi-\tilde{\xi}|} \leq \ell_{j} .
$$


$\left(\mathbf{A}_{\mathbf{2}}\right)$ For $j=1, \cdots, n$, the delay kernels $K_{j}():. \mathbb{R}_{+} \rightarrow \mathbb{R}_{+}$are bump functions satisfying

$$
\int_{0}^{+\infty} K_{j}(s) d s=1 .
$$

$\left(\mathbf{A}_{\mathbf{3}}\right)$ There exists a positive constant $\mathbb{M}_{j}$ such that for $j=1, \cdots, n$, we have

$$
\left|f_{j}(.)\right| \leq \mathbb{M}_{j}
$$

Remark 3.2. Under Assumptions $\left(\mathbf{A}_{\mathbf{1}}\right)$ and $\left(\mathbf{A}_{\mathbf{2}}\right)$, the existence of solutions of the system (2) is ensured as it is explained in 37 .

Let $y^{*}$ be an equilibrium point of the system (2). By a simple transformation $z_{i}(t)=y_{i}(t)-y^{*} \in \mathbb{R}$, we can shift the equilibrium point to the origin. Then, the system (2) can be rewritten as follows:

$$
\begin{aligned}
\frac{d^{2} z_{i}(t)}{d t^{2}}= & -a_{i} \frac{d z_{i}(t)}{d t}-b_{i} z_{i}(t)+\sum_{j=1}^{n} c_{i j} F_{j}\left(z_{j}(t)\right)+\sum_{j=1}^{n} d_{i j} F_{j}\left(z_{j}\left(t-\tau_{j}(t)\right)\right) \\
& +\sum_{j=1}^{n} \eta_{i j} \int_{-\infty}^{t} K(t-s) F_{j}\left(z_{j}(s)\right) d s+\sum_{j=1}^{n} e_{i j} F_{j}\left(\dot{z}_{j}\left(t-\tau_{j}(t)\right)\right) \\
& +\bigwedge_{j=1}^{n} \alpha_{i j} F_{j}\left(z_{j}\left(t-\tau_{j}(t)\right)\right)+\bigvee_{j=1}^{n} \beta_{i j} F_{j}\left(z_{j}\left(t-\tau_{j}(t)\right)\right) \\
& +\bigwedge_{j=1}^{n} \gamma_{i j} F_{j}\left(\dot{z}_{j}\left(t-\tau_{j}(t)\right)\right)+\bigvee_{j=1}^{n} \delta_{i j} F_{j}\left(\dot{z}_{j}\left(t-\tau_{j}(t)\right)\right)
\end{aligned}
$$

where $F_{j}\left(z_{j}\right)=f_{j}\left(z_{j}+y^{*}\right)-f_{j}\left(y_{j}^{*}\right)$. The initial conditions of the system 4 are given by

$$
\begin{aligned}
z_{i}(s) & =\check{\varphi}_{i}(s)-y^{*}=\varphi(s), \\
\frac{d z_{i}(s)}{d t} & =\check{\psi}_{i}(s)-y^{*}=\psi(s), \quad s \in[-\tau, 0], i=1, \cdots, n
\end{aligned}
$$

For $\zeta_{i} \in \mathbb{R}$, letting $p_{i}(t)=\frac{d z_{i}(t)}{d t}+\zeta_{i} z_{i}(t)$ for $i=1, \cdots, n$, then the system 4 with the control variables can be rewritten as

$$
\left\{\begin{aligned}
\frac{d z_{i}(t)}{d t}= & -\zeta_{i} z_{i}(t)+p_{i}(t)+\hat{Q}_{i}(t) \\
\frac{d p_{i}(t)}{d t}= & -\bar{a}_{i} p_{i}(t)+\bar{b}_{i} z_{i}(t)+\sum_{j=1}^{n} c_{i j} F_{j}\left(z_{j}(t)\right)+\sum_{j=1}^{n} d_{i j} F_{j}\left(z_{j}\left(t-\tau_{j}(t)\right)\right) \\
& +\sum_{j=1}^{n} \eta_{i j} \int_{-\infty}^{t} K(t-s) f_{j}\left(z_{j}(s)\right) d s+\sum_{j=1}^{n} e_{i j} f_{j}\left(\dot{z}_{j}\left(t-\tau_{j}(t)\right)\right) \\
& +\bigwedge_{j=1}^{n} \alpha_{i j} F_{j}\left(z_{j}\left(t-\tau_{j}(t)\right)\right)+\bigvee_{j=1}^{n} \beta_{i j} F_{j}\left(z_{j}\left(t-\tau_{j}(t)\right)\right) \\
& +\bigwedge_{j=1}^{n} \gamma_{i j} F_{j}\left(\dot{z}_{j}\left(t-\tau_{j}(t)\right)\right)+\bigvee_{j=1}^{n} \delta_{i j} F_{j}\left(\dot{z}_{j}\left(t-\tau_{j}(t)\right)\right)+\check{Q}_{i}(t), \quad i=1, \ldots, n
\end{aligned}\right.
$$

where $\bar{a}_{i}=a_{i}-\zeta_{i}, \bar{b}_{i}=\zeta_{i} \bar{a}_{i}-b_{i}$ and $\hat{Q}=\left(\hat{Q}_{1}, \cdots, \hat{Q}_{n}\right), \check{Q}=\left(\check{Q}_{1}, \cdots, \check{Q}_{n}\right)$ are the control variables and the initial conditions become

$$
\begin{aligned}
& z_{i}(s)=\varphi(s) \\
& p_{i}(t)=\zeta_{i} \varphi(s)+\psi(s), s \in[-\tau, 0], i=1, \cdots, n .
\end{aligned}
$$


Definition 3. The system $\sqrt{5}$ is finite-time stabilizable if under a suitable designed feedback controls $\hat{Q}_{i}(t)$ and $\breve{Q}_{i}(t)$ there exists $\mathbf{T}$ dependent on the initial conditions $(6)$ such that the closed-loop system is finite-time stable. Moreover, the system (5) is fixed-time stabilizable if:

(1) it is finite-time stabilizable;

(2) the settling-time functional $\mathbf{T}$ is upper bounded by a constant $\mathbf{T}^{\max } \geq 0$, i.e., $\mathbf{T} \leq \mathbf{T}^{\max }$.

Remark 3.3. The stabilization of NNs has been developed to solve problems of classification, prediction, categorisation, optimization, recognition of forms, associative memory secure communication [12, 30, 38, 39]. In these fields, sometimes it is necessary to complete certain tasks within a finite time or fixed time. Contrary to the finite-time stabilization, the settling-time for fixed-time stabilization is independent on the initial conditions. In some areas, such as pattern recognition secure communication, the initial values are mostly hard to be acquired [40], so fixed-time stabilization is more accurate and applicable than finite-time stabilization. Thus, the pre-specified settling-time can be obtained by properly adjust the control parameters.

Lemma 6. 41 For $j=1, \cdots, n$, let $z_{j}, \tilde{z}_{j}$ be two states of the system (5) and $F_{j}: \mathbb{R} \rightarrow \mathbb{R}$ be continuous functions, then we have

$$
\begin{aligned}
\left|\bigwedge_{j=1}^{n} \alpha_{i j} F_{j}\left(z_{j}\right)-\bigwedge_{j=1}^{n} \alpha_{i j} F_{j}\left(\tilde{z}_{j}\right)\right| & \leq \sum_{j=1}^{n}\left|\alpha_{i j}\right|\left|F_{j}\left(z_{j}\right)-F_{j}\left(\tilde{z}_{j}\right)\right| \\
\left|\bigvee_{j=1}^{n} \beta_{i j} F_{j}\left(z_{j}\right)-\bigvee_{j=1}^{n} \beta_{i j} F_{j}\left(\tilde{z}_{j}\right)\right| & \leq \sum_{j=1}^{n}\left|\beta_{i j}\right|\left|F_{j}\left(z_{j}\right)-F_{j}\left(\tilde{z}_{j}\right)\right|
\end{aligned}
$$

\section{Main results}

In this section, the fixed-time stabilization of FNTINNs with time-varying delay is investigated. By using the fixed-time stability results in Section 2, two different feedback control laws are designed to ensure the fixed-time stabilization of FNTINNs, which includes and extends the earlier works.

Theorem 1. Assume that $\left(\mathbf{A}_{\mathbf{1}}\right)$ and $\left(\mathbf{A}_{\mathbf{2}}\right)$ hold and the time-varying delay $\tau_{j}(t)$ is known. The system (5) is fixed-time stabilized under the following feedback control law

$$
\begin{aligned}
\hat{Q}_{i}(t)= & -h_{i 1} z_{i}(t)-h_{i 2} \operatorname{sign}\left(z_{i}(t)\right)\left|z_{i}(t)\right|^{\kappa}-h_{i 3} \operatorname{sign}\left(z_{i}(t)\right)\left|z_{i}(t)\right|^{\mu} \\
\check{Q}_{i}(t)= & -m_{i 1} p_{i}(t)-m_{i 2} \operatorname{sign}\left(p_{i}(t)\right)\left|p_{i}(t)\right|^{\kappa}-m_{i 3} \operatorname{sign}\left(p_{i}(t)\right)\left|p_{i}(t)\right|^{\mu} \\
& -\sum_{j=1}^{n} \eta_{i j} \int_{-\infty}^{t} K(t-s) F_{j}\left(z_{j}(s)\right) d s-\sum_{j=1}^{n} d_{i j} F_{j}\left(z_{j}\left(t-\tau_{j}(t)\right)\right) \\
& -\sum_{j=1}^{n} e_{i j} F_{j}\left(\dot{z}_{j}\left(t-\tau_{j}(t)\right)\right)-\bigwedge_{j=1}^{n} \alpha_{i j} F_{j}\left(z_{j}\left(t-\tau_{j}(t)\right)\right)-\bigvee_{j=1}^{n} \beta_{i j} F_{j}\left(z_{j}\left(t-\tau_{j}(t)\right)\right) \\
& -\bigwedge_{j=1}^{n} \gamma_{i j} F_{j}\left(\dot{z}_{j}\left(t-\tau_{j}(t)\right)\right)-\bigvee_{j=1}^{n} \delta_{i j} F_{j}\left(\dot{z}_{j}\left(t-\tau_{j}(t)\right)\right)
\end{aligned}
$$

where

$$
\begin{gathered}
h_{i 1}>-\zeta_{i}+\frac{1}{2}+\frac{\left|\bar{b}_{i}\right|}{2}+\sum_{j=1}^{n} \frac{\left|c_{j i}\right|}{2} \ell_{i} ; \\
m_{i 1}>-\bar{a}_{i}+\frac{1}{2}+\frac{\left|\bar{b}_{i}\right|}{2}+\sum_{j=1}^{n} \frac{\left|c_{i j}\right|}{2} \ell_{j} ; \\
h_{i 2}>0 ; \quad h_{i 3}>0 ; \quad m_{i 2}>0, m_{i 3}>0,0<\kappa<1 ; \quad \mu>1 .
\end{gathered}
$$


Taking

$$
\begin{aligned}
& \rho_{i 1}=\zeta_{i}-\frac{1}{2}+h_{i 1}-\frac{\left|\bar{b}_{i}\right|}{2}-\sum_{j=1}^{n} \frac{\left|c_{j i}\right|}{2} \ell_{i} ; \quad \rho_{i 2}=-\frac{1}{2}+\bar{a}_{i}+m_{i 1}-\frac{\left|\bar{b}_{i}\right|}{2}-\sum_{j=1}^{n} \frac{\left|c_{i j}\right|}{2} \ell_{j} ; \\
& \underline{a}=2 \min \left\{\min _{1 \leq i \leq n}\left\{\rho_{i 1}\right\}, \min _{1 \leq i \leq n}\left\{\rho_{i 2}\right\}\right\} ; \quad \underline{b}=\min \left\{\min _{1 \leq i \leq n}\left\{h_{i 2}\right\}, \min _{1 \leq i \leq n}\left\{m_{i 2}\right\}\right\} 2^{\frac{\kappa+1}{2}} \\
& \underline{c}=\min \left\{\min _{1 \leq i \leq n}\left\{h_{i 3}\right\}, \min _{1 \leq i \leq n}\left\{m_{i 3}\right\}\right\} 2^{\frac{\mu+1}{2}} n^{\frac{1-\mu}{2}}
\end{aligned}
$$

the settling-time $\mathbf{T}$ satisfies

$$
\mathbf{T} \leq \mathbf{T}_{5}^{\max }=\frac{2}{\underline{a}(1-\kappa)} \log \left(\frac{\underline{b}+\underline{a}}{\underline{b}}\right)+\frac{2}{\underline{a}(\mu-1)} \log \left(\frac{\underline{c}+\underline{a}}{\underline{c}}\right) .
$$

Proof. With the feedback control law (7), the closed-loop system (5)-(7) leads to the following delay-free system

$$
\begin{aligned}
\frac{d z_{i}(t)}{d t}= & -\zeta_{i} z_{i}(t)+p_{i}(t)-h_{i 1} z_{i}(t)-h_{i 2} \operatorname{sign}\left(z_{i}(t)\right)\left|z_{i}(t)\right|^{\kappa}-h_{i 3} \operatorname{sign}\left(z_{i}(t)\right)\left|z_{i}(t)\right|^{\mu} \\
\frac{d p_{i}(t)}{d t}= & -\bar{a}_{i} p_{i}(t)+\bar{b}_{i} z_{i}(t)+\sum_{j=1}^{n} c_{i j} F_{j}\left(z_{j}(t)\right) \\
& -m_{i 1} p_{i}(t)-m_{i 2} \operatorname{sign}\left(p_{i}(t)\right)\left|p_{i}(t)\right|^{\kappa}-m_{i 3} \operatorname{sign}\left(p_{i}(t)\right)\left|p_{i}(t)\right|^{\mu} .
\end{aligned}
$$

Let us choose the following Lyapunov function candidate:

$$
V(t)=\frac{1}{2} \sum_{i=1}^{n} z_{i}^{2}(t)+\frac{1}{2} \sum_{i=1}^{n} p_{i}^{2}(t)
$$

By using Assumption $\left(\mathbf{A}_{\mathbf{1}}\right)$, we have that the time derivative of 13 along the trajectories of the system 12 . satisfies

$$
\begin{aligned}
\frac{d V(t)}{d t} \leq & \sum_{i=1}^{n}\left[-\zeta_{i} z_{i}^{2}(t)+p_{i}(t) z_{i}(t)-h_{i 1} z_{i}^{2}(t)-h_{i 2}\left|z_{i}(t)\right|^{\kappa+1}-h_{i 3}\left|z_{i}(t)\right|^{\mu+1}\right] \\
& +\sum_{i=1}^{n}\left[-\bar{a}_{i} p_{i}^{2}(t)+\left|\bar{b}_{i}\right|\left|z_{i}(t)\right|\left|p_{i}(t)\right|+\sum_{j=1}^{n}\left|c_{i j}\right| \ell_{j}\left|z_{j}(t)\right|\left|p_{i}(t)\right|\right. \\
& \left.-m_{i 1} p_{i}^{2}(t)-m_{i 2}\left|p_{i}(t)\right|^{\kappa+1}-m_{i 3}\left|p_{i}(t)\right|^{\mu+1}\right] .
\end{aligned}
$$

Since, we have $2 x y \leq x^{2}+y^{2}$ for all $x, y \in \mathbb{R}^{+}$, it follows that

$$
\left|\bar{b}_{i}\right|\left|z_{i}(t)\right|\left|p_{i}(t)\right| \leq\left|\bar{b}_{i}\right|\left(\frac{p_{i}^{2}(t)}{2}+\frac{z_{i}^{2}(t)}{2}\right)
$$

and

$$
\begin{aligned}
\sum_{i=1}^{n} \sum_{j=1}^{n}\left|c_{i j}\right| \ell_{j}\left|z_{j}(t)\right|\left|p_{i}(t)\right| & \leq \sum_{i=1}^{n} \sum_{j=1}^{n} \frac{\left|c_{i j}\right|}{2} \ell_{j} z_{j}^{2}(t)+\sum_{i=1}^{n} \sum_{j=1}^{n} \frac{\left|c_{i j}\right|}{2} \ell_{j} p_{i}^{2}(t) \\
& \leq \sum_{i=1}^{n} \sum_{j=1}^{n} \frac{\left|c_{j i}\right|}{2} \ell_{i} z_{i}^{2}(t)+\sum_{i=1}^{n} \sum_{j=1}^{n} \frac{\left|c_{i j}\right|}{2} \ell_{j} p_{i}^{2}(t) .
\end{aligned}
$$


From (14)-(16), we obtain

$$
\begin{aligned}
\frac{d V(t)}{d t} \leq & \sum_{i=1}^{n}\left[-\zeta_{i} z_{i}^{2}(t)+\frac{p_{i}^{2}(t)}{2}+\frac{z_{i}^{2}(t)}{2}-h_{i 1} z_{i}^{2}(t)-h_{i 2}\left|z_{i}(t)\right|^{\kappa+1}-h_{i 3}\left|z_{i}(t)\right|^{\mu+1}\right] \\
& +\sum_{i=1}^{n}\left[-\bar{a}_{i} p_{i}^{2}(t)+\left|\bar{b}_{i}\right|\left(\frac{p_{i}^{2}(t)}{2}+\frac{z_{i}^{2}(t)}{2}\right)\right. \\
& \left.+\sum_{j=1}^{n} \frac{\left|c_{j i}\right|}{2} \ell_{i} z_{i}^{2}(t)+\sum_{j=1}^{n} \frac{\left|c_{i j}\right|}{2} \ell_{j} p_{i}^{2}(t)-m_{i 1} p_{i}^{2}(t)-m_{i 2}\left|p_{i}(t)\right|^{\kappa+1}-m_{i 3}\left|p_{i}(t)\right|^{\mu+1}\right] \\
\leq & \sum_{i=1}^{n}\left[-\zeta_{i}+\frac{1}{2}-h_{i 1}+\frac{\left|\bar{b}_{i}\right|}{2}+\sum_{j=1}^{n} \frac{\left|c_{j i}\right|}{2} \ell_{i}\right] z_{i}^{2}(t)-\sum_{i=1}^{n} h_{i 2}\left|z_{i}(t)\right|^{\kappa+1} \\
& -\sum_{i=1}^{n} h_{i 3}\left|z_{i}(t)\right|^{\mu+1}+\sum_{i=1}^{n}\left[\frac{1}{2}-\bar{a}_{i}+\frac{\left|\bar{b}_{i}\right|}{2}+\sum_{j=1}^{n} \frac{\left|c_{i j}\right|}{2} \ell_{j}-m_{i 1}\right] p_{i}^{2}(t) \\
& -\sum_{i=1}^{n} m_{i 2}\left|p_{i}(t)\right|^{\kappa+1}-\sum_{i=1}^{n} m_{i 3}\left|p_{i}(t)\right|^{\mu+1} \\
\leq & \sum_{i=1}^{n}-\rho_{i 1} z_{i}^{2}(t)+\sum_{i=1}^{n}-\rho_{i 2} p_{i}^{2}(t)-\sum_{i=1}^{n} h_{i 2}\left|z_{i}(t)\right|^{\kappa+1} \\
& -\sum_{i=1}^{n} h_{i 3}\left|z_{i}(t)\right|^{\mu+1}-\sum_{i=1}^{n} m_{i 2}\left|p_{i}(t)\right|^{\kappa+1}-\sum_{i=1}^{n} m_{i 3}\left|p_{i}(t)\right|^{\mu+1}
\end{aligned}
$$

where

$$
\rho_{i 1}=\zeta_{i}-\frac{1}{2}+h_{i 1}-\frac{\left|\bar{b}_{i}\right|}{2}-\sum_{j=1}^{n} \frac{\left|c_{j i}\right|}{2} \ell_{i}>0
$$

and

$$
\rho_{i 2}=-\frac{1}{2}+\bar{a}_{i}+m_{i 1}-\frac{\left|\bar{b}_{i}\right|}{2}-\sum_{j=1}^{n} \frac{\left|c_{i j}\right|}{2} \ell_{j}>0 .
$$

Then, it yields

$$
\begin{aligned}
& \frac{d V(t)}{d t} \leq-\min _{1 \leq i \leq n}\left\{\rho_{i 1}\right\} \sum_{i=1}^{n} z_{i}^{2}(t)-\min _{1 \leq i \leq n}\left\{\rho_{i 2}\right\} \sum_{i=1}^{n} p_{i}^{2}(t) \\
& -\min _{1 \leq i \leq n}\left\{h_{i 2}\right\} \sum_{i=1}^{n}\left|z_{i}(t)\right|^{\kappa+1}-\min _{1 \leq i \leq n}\left\{h_{i 3}\right\} \sum_{i=1}^{n}\left|z_{i}(t)\right|^{\mu+1} \\
& -\min _{1 \leq i \leq n}\left\{m_{i 2}\right\} \sum_{i=1}^{n}\left|p_{i}(t)\right|^{\kappa+1}-\min _{1 \leq i \leq n}\left\{m_{i 3}\right\} \sum_{i=1}^{n}\left|p_{i}(t)\right|^{\mu+1} \\
& \leq-\min \left\{\min _{1 \leq i \leq n}\left\{\rho_{i 1}\right\}, \min _{1 \leq i \leq n}\left\{\rho_{i 2}\right\}\right\}\left(\sum_{i=1}^{n} z_{i}^{2}(t)+\sum_{i=1}^{n} p_{i}^{2}(t)\right) \\
& -\min \left\{\min _{1 \leq i \leq n}\left\{h_{i 2}\right\}, \min _{1 \leq i \leq n}\left\{m_{i 2}\right\}\right\}\left(\sum_{i=1}^{n}\left|z_{i}(t)\right|^{\kappa+1}+\sum_{i=1}^{n}\left|p_{i}(t)\right|^{\kappa+1}\right) \\
& -\min \left\{\min _{1 \leq i \leq n}\left\{h_{i 3}\right\}, \min _{1 \leq i \leq n}\left\{m_{i 3}\right\}\right\}\left(\sum_{i=1}^{n}\left|z_{i}(t)\right|^{\mu+1}+\sum_{i=1}^{n}\left|p_{i}(t)\right|^{\mu+1}\right) \text {. }
\end{aligned}
$$


Since we have $0<\kappa<1$, it follows from Lemma 5 that

$$
\begin{aligned}
\sum_{i=1}^{n}\left|z_{i}(t)\right|^{\kappa+1}+\sum_{i=1}^{n}\left|p_{i}(t)\right|^{\kappa+1} & =\sum_{i=1}^{n}\left(z_{i}^{2}(t)\right)^{\frac{\kappa+1}{2}}+\sum_{i=1}^{n}\left(p_{i}^{2}(t)\right)^{\frac{\kappa+1}{2}} \\
& \geq\left(\sum_{i=1}^{n} z_{i}^{2}(t)\right)^{\frac{\kappa+1}{2}}+\left(\sum_{i=1}^{n} p_{i}^{2}(t)\right)^{\frac{\kappa+1}{2}} \\
& \geq\left(\sum_{i=1}^{n} z_{i}^{2}(t)+\sum_{i=1}^{n} p_{i}^{2}(t)\right)^{\frac{\kappa+1}{2}} .
\end{aligned}
$$

On the other hand, we have $\mu>1$ then it follows from Lemma 5 that

$$
\begin{aligned}
\sum_{i=1}^{n}\left|z_{i}(t)\right|^{\mu+1}+\sum_{i=1}^{n}\left|p_{i}(t)\right|^{\mu+1} & =\sum_{i=1}^{n}\left(z_{i}^{2}(t)\right)^{\frac{\mu+1}{2}}+\sum_{i=1}^{n}\left(p_{i}^{2}(t)\right)^{\frac{\mu+1}{2}} \\
& \geq n^{\frac{1-\mu}{2}}\left(\left(\sum_{i=1}^{n} z_{i}^{2}(t)\right)^{\frac{\mu+1}{2}}+\left(\sum_{i=1}^{n} p_{i}^{2}(t)\right)^{\frac{\mu+1}{2}}\right) \\
& \geq 2^{\frac{1-\mu}{2}} n^{\frac{1-\mu}{2}}\left(\sum_{i=1}^{n} z_{i}^{2}(t)+\sum_{i=1}^{n} p_{i}^{2}(t)\right)^{\frac{\mu+1}{2}} .
\end{aligned}
$$

From $18-20$, we have

$$
\begin{aligned}
\frac{d V(t)}{d t} \leq & -2 \min \left\{\min _{1 \leq i \leq n}\left\{\rho_{i 1}\right\}, \min _{1 \leq i \leq n}\left\{\rho_{i 2}\right\}\right\} V(t) \\
& -\min \left\{\min _{1 \leq i \leq n}\left\{h_{i 2}\right\}, \min _{1 \leq i \leq n}\left\{m_{i 2}\right\}\right\} 2^{\frac{\kappa+1}{2}} V^{\frac{\kappa+1}{2}}(t) \\
& -\min \left\{\min _{1 \leq i \leq n}\left\{h_{i 2}\right\}, \min _{1 \leq i \leq n}\left\{m_{i 2}\right\}\right\} 2^{\frac{\mu+1}{2}} 2^{\frac{1-\mu}{2}} n^{\frac{1-\mu}{2}} V^{\frac{\mu+1}{2}}(t) \\
\leq & -2 \min \left\{\min _{1 \leq i \leq n}\left\{\rho_{i 1}\right\}, \min _{1 \leq i \leq n}\left\{\rho_{i 2}\right\}\right\} V(t) \\
& -\min \left\{\min _{1 \leq i \leq n}\left\{h_{i 2}\right\}, \min _{1 \leq i \leq n}\left\{m_{i 2}\right\}\right\} 2^{\frac{\kappa+1}{2}} V^{\frac{\kappa+1}{2}}(t) \\
& -\min \left\{\min _{1 \leq i \leq n}\left\{h_{i 2}\right\}, \min _{1 \leq i \leq n}\left\{m_{i 2}\right\}\right\} 2 n^{\frac{1-\mu}{2}} V^{\frac{\mu+1}{2}}(t) .
\end{aligned}
$$

Let

$$
\begin{aligned}
& \underline{a}=2 \min \left\{\min _{1 \leq i \leq n}\left\{\rho_{i 1}\right\}, \min _{1 \leq i \leq n}\left\{\rho_{i 2}\right\}\right\} \\
& \underline{b}=\min \left\{\min _{1 \leq i \leq n}\left\{h_{i 2}\right\}, \min _{1 \leq i \leq n}\left\{m_{i 2}\right\}\right\} 2^{\frac{\kappa+1}{2}} \\
& \underline{c}=\min \left\{\min _{1 \leq i \leq n}\left\{h_{i 3}\right\}, \min _{1 \leq i \leq n}\left\{m_{i 3}\right\}\right\} 2 n^{\frac{1-\mu}{2}}
\end{aligned}
$$

from (21) we obtain

$$
\frac{d V(t)}{d t} \leq-\underline{a} V(t)-\underline{b} V^{\frac{\kappa+1}{2}}(t)-\underline{c} V^{\frac{\mu+1}{2}}(t) .
$$

According to Lemma 4 the system (5) is fixed-time stable and the settling-time satisfies (11). 
Remark 4.1. On the one hand, the class of FNTINN with mixed delays have more complex dynamic behaviors compared with traditional NNs [26, 28, 42, 43]. On the other hand, it is delicate to design a Lyapunov functional satisfying the derivative condition for fixed-time stabilization of time-delay systems [32]. In our article, the stabilization of FNTINN with mixed delays is investigated which renders the results more general compared with the above-mentioned ones.

Remark 4.2. Given that $\alpha>0, \log (1+\alpha)<\alpha$, we have

$$
\frac{1}{\underline{a}(1-\kappa)} \log \left(\frac{\underline{b}+\underline{a}}{\underline{b}}\right)+\frac{1}{\underline{a}(\mu-1)} \log \left(\frac{\underline{c}+\underline{a}}{\underline{c}}\right)<\frac{1}{\underline{b}(1-\kappa)}+\frac{1}{\underline{c}(\mu-1)} .
$$

It implies that $\mathbf{T}_{5}^{\max }<\mathbf{T}_{2}^{\max }$. Consequently, the upper settling-time obtained in Theorem 1 is much smaller than the upper settling-time obtained in [44, 45, 46].

Corollary 1. Assume that $\left(\mathbf{A}_{\mathbf{1}}\right)$ and $\left(\mathbf{A}_{\mathbf{2}}\right)$ hold and the conditions 8 - $-(10)$ are satisfied. Then the system (5) is fixed-time stabilizable under the feedback control law (7). Moreover, the settling-time $\mathbf{T}$ satisfies

$$
\mathbf{T} \leq \mathbf{T}_{6}^{\max }=\frac{2}{\underline{b}(1-\kappa)}+\frac{2}{\underline{c}(\mu-1)}
$$

where

$$
\underline{b}=\min \left\{\min _{1 \leq i \leq n}\left\{h_{i 2}\right\}, \min _{1 \leq i \leq n}\left\{m_{i 2}\right\}\right\} 2^{\frac{\kappa+1}{2}} ; \underline{c}=\min \left\{\min _{1 \leq i \leq n}\left\{h_{i 3}\right\}, \min _{1 \leq i \leq n}\left\{m_{i 3}\right\}\right\} 2 n^{\frac{1-\mu}{2}} .
$$

Proof. From 22, we have

$$
\begin{aligned}
\frac{d V(t)}{d t} & \leq-\underline{a} V(t)-\underline{b} V^{\frac{\kappa+1}{2}}(t)-\underline{c} V^{\frac{\mu+1}{2}}(t) \\
& \leq-\underline{b} V^{\frac{\kappa+1}{2}}(t)-\underline{c} V^{\frac{\mu+1}{2}}(t) .
\end{aligned}
$$

Then the result directly follows from Lemma 1 .

Corollary 2. Assume that $\left(\mathbf{A}_{\mathbf{1}}\right)$ and $\left(\mathbf{A}_{\mathbf{2}}\right)$ hold and the conditions (8)-(10) are satisfied. Then the system (5) is fixed-time stabilizable under the feedback control law (7). Moreover, the settling-time $\mathbf{T}$ satisfies

$$
\mathbf{T} \leq \mathbf{T}_{7}^{\max }=\frac{1}{\underline{b}}\left(\frac{\underline{b}}{\underline{c}}\right)^{\frac{1-\kappa}{\mu-\kappa}}\left(\frac{2}{1-\kappa}+\frac{2}{\mu-1}\right) .
$$

Proof. From 22, we have

$$
\begin{aligned}
\frac{d V(t)}{d t} & \leq-\underline{a} V(t)-\underline{b} V^{\frac{\kappa+1}{2}}(t)-\underline{c} V^{\frac{\mu+1}{2}}(t) \\
& \leq-\underline{b} V^{\frac{\kappa+1}{2}}(t)-\underline{c} V^{\frac{\mu+1}{2}}(t) .
\end{aligned}
$$

Then the result directly follows from Lemma 2

In Theorem 1, by designing a special fixed-time controller, we achieved the fixed-time stabilization of the system (5). However, $\check{Q}_{i}$ in (7) is a delay dependent feedback control, which is not suitable for real-world applications. Thus, we seek to obtain a fixed-time controller that is more suitable in practice and able to stabilize in fixed-time the FNTINNs (5). To this end, we need to impose the boundedness of our activation functions and we have the following result:

Theorem 2. Assume that $\left(\mathbf{A}_{\mathbf{1}}\right)-\left(\mathbf{A}_{\mathbf{3}}\right)$ hold and the conditions (8)-10) are satisfied. Let

$$
\varpi>2 \sum_{j=1}^{n} \mathbb{M}_{j}\left(\left|d_{i j}\right|+\left|\eta_{i j}\right|+\left|e_{i j}\right|+\left|\alpha_{i j}\right|+\left|\beta_{i j}\right|+\left|\gamma_{i j}\right|+\left|\delta_{i j}\right|\right)
$$


then the system (5) is fixed-time stabilizable under the feedback control law

$$
\begin{aligned}
& \hat{Q}_{i}(t)=-h_{i 1} z_{i}(t)-h_{i 2} \operatorname{sign}\left(z_{i}(t)\right)\left|z_{i}(t)\right|^{\kappa}-h_{i 3} \operatorname{sign}\left(z_{i}(t)\right)\left|z_{i}(t)\right|^{\mu} \\
& \check{Q}_{i}(t)=-m_{i 1} p_{i}(t)-m_{i 2} \operatorname{sign}\left(p_{i}(t)\right)\left|p_{i}(t)\right|^{\kappa}-m_{i 3} \operatorname{sign}\left(p_{i}(t)\right)\left|p_{i}(t)\right|^{\mu}-\varpi \operatorname{sign}\left(p_{i}(t)\right), \quad i=1, \ldots, n
\end{aligned}
$$

and the settling-time is bounded from above by $\mathbf{T}_{5}^{\max }$.

Proof. Let us choose the following Lyapunov function candidate:

$$
V(t)=\frac{1}{2} \sum_{i=1}^{n} z_{i}^{2}(t)+\frac{1}{2} \sum_{i=1}^{n} p_{i}^{2}(t) .
$$

The time derivative of (29) along the trajectories of the system (5) satisfies

$$
\begin{aligned}
& \frac{d V(t)}{d t}=\sum_{i=1}^{n} z_{i}(t) \frac{d z_{i}(t)}{d t}+\sum_{i=1}^{n} p_{i}(t) \frac{d p_{i}(t)}{d t} \\
& =\sum_{i=1}^{n}\left[-\zeta_{i} z_{i}^{2}(t)+p_{i}(t) z_{i}(t)-h_{i 1} z_{i}^{2}(t)-h_{i 2}\left|z_{i}(t)\right|^{\kappa+1}-h_{i 3}\left|z_{i}(t)\right|^{\mu+1}\right] \\
& +\sum_{i=1}^{n}\left[-\bar{a}_{i} p_{i}^{2}(t)+\bar{b}_{i} z_{i}(t) p_{i}(t)+\sum_{j=1}^{n} c_{i j} F_{j}\left(z_{j}(t)\right) p_{i}(t)+\sum_{j=1}^{n} d_{i j} F_{j}\left(z_{j}\left(t-\tau_{j}(t)\right)\right) p_{i}(t)\right. \\
& +\sum_{j=1}^{n} \eta_{i j} \int_{-\infty}^{t} K(t-s) F_{j}\left(z_{j}(s)\right) d s p_{i}(t)+\sum_{j=1}^{n} e_{i j} F_{j}\left(\dot{z}_{j}\left(t-\tau_{j}(t)\right)\right) p_{i}(t) \\
& +\bigwedge_{j=1}^{n} \alpha_{i j} F_{j}\left(z_{j}\left(t-\tau_{j}(t)\right)\right) p_{i}(t)+\bigvee_{j=1}^{n} \beta_{i j} F_{j}\left(z_{j}\left(t-\tau_{j}(t)\right)\right) p_{i}(t) \\
& +\bigwedge_{j=1}^{n} \gamma_{i j} F_{j}\left(\dot{z}_{j}\left(t-\tau_{j}(t)\right)\right) p_{i}(t)+\bigvee_{j=1}^{n} \delta_{i j} F_{j}\left(\dot{z}_{j}\left(t-\tau_{j}(t)\right)\right) p_{i}(t) \\
& \left.-m_{i 1} p_{i}^{2}(t)-m_{i 2}\left|p_{i}(t)\right|^{\kappa+1}-m_{i 3}\left|p_{i}(t)\right|^{\mu+1}-\varpi\left|p_{i}(t)\right|\right] \\
& \leq \sum_{i=1}^{n}\left[-\zeta_{i} z_{i}^{2}(t)+p_{i}(t) z_{i}(t)-h_{i 1} z_{i}^{2}(t)-h_{i 2}\left|z_{i}(t)\right|^{\kappa+1}-h_{i 3}\left|z_{i}(t)\right|^{\mu+1}\right] \\
& +\sum_{i=1}^{n}\left[-\bar{a}_{i} p_{i}^{2}(t)+\left|\bar{b}_{i}\right| z_{i}(t) p_{i}(t)+\sum_{j=1}^{n}\left|c_{i j}\right| \ell_{j}\left|z_{j}(t)\right|\left|p_{i}(t)\right|+\sum_{j=1}^{n}\left|d_{i j}\right|\left|F_{j}\left(z_{j}\left(t-\tau_{j}(t)\right)\right)\right|\left|p_{i}(t)\right|\right. \\
& +\sum_{j=1}^{n}\left|\eta_{i j}\right| \int_{-\infty}^{t} K(t-s)\left|F_{j}\left(z_{j}(s)\right)\right| d s\left|p_{i}(t)\right|+\sum_{j=1}^{n}\left|e_{i j}\right|\left|F_{j}\left(\dot{z}_{j}\left(t-\tau_{j}(t)\right)\right)\right|\left|p_{i}(t)\right| \\
& +\left|\bigwedge_{j=1}^{n} \alpha_{i j} F_{j}\left(z_{j}\left(t-\tau_{j}(t)\right)\right)\right|\left|p_{i}(t)\right|+\left|\bigvee_{j=1}^{n} \beta_{i j} F_{j}\left(z_{j}\left(t-\tau_{j}(t)\right)\right)\right|\left|p_{i}(t)\right| \\
& +\left|\bigwedge_{j=1}^{n} \gamma_{i j} F_{j}\left(\dot{z}_{j}\left(t-\tau_{j}(t)\right)\right)\right|\left|p_{i}(t)\right|+\left|\bigvee_{j=1}^{n} \delta_{i j} F_{j}\left(\dot{z}_{j}\left(t-\tau_{j}(t)\right)\right)\right|\left|p_{i}(t)\right| \\
& \left.-m_{i 1} p_{i}^{2}(t)-m_{i 2}\left|p_{i}(t)\right|^{\kappa+1}-m_{i 3}\left|p_{i}(t)\right|^{\mu+1}-\varpi\left|p_{i}(t)\right|\right] .
\end{aligned}
$$

From $\left(\mathbf{A}_{\mathbf{2}}\right)$ and $\left(\mathbf{A}_{\mathbf{3}}\right)$, we have

$$
\sum_{j=1}^{n}\left|\eta_{i j}\right| \int_{-\infty}^{t} K(t-s)\left|F_{j}\left(z_{j}(s)\right)\right| d s\left|p_{i}(t)\right| \leq \sum_{j=1}^{n} 2\left|\eta_{i j}\right| \mathbb{M}_{j}\left|p_{i}(t)\right| .
$$


From Lemma 6 and $\left(\mathbf{A}_{\mathbf{3}}\right)$, we have

$$
\begin{aligned}
\left|\bigwedge_{j=1}^{n} \alpha_{i j} F_{j}\left(z_{j}\left(t-\tau_{j}(t)\right)\right)\right| \leq \sum_{j=1}^{n}\left|\alpha_{i j}\right|\left|F_{j}\left(z_{j}\left(t-\tau_{j}(t)\right)\right)\right| \leq \sum_{j=1}^{n} 2\left|\alpha_{i j}\right| \mathbb{M}_{j} \\
\left|\bigvee_{j=1}^{n} \beta_{i j} F_{j}\left(z_{j}\left(t-\tau_{j}(t)\right)\right)\right| \leq \sum_{j=1}^{n}\left|\beta_{i j}\right|\left|F_{j}\left(z_{j}\left(t-\tau_{j}(t)\right)\right)\right| \leq \sum_{j=1}^{n} 2\left|\beta_{i j}\right| \mathbb{M}_{j}, \\
\left|\bigwedge_{j=1}^{n} \gamma_{i j} F_{j}\left(\dot{z}_{j}\left(t-\tau_{j}(t)\right)\right)\right| \leq \sum_{j=1}^{n}\left|\gamma_{i j}\right|\left|F_{j}\left(\dot{z}_{j}\left(t-\tau_{j}(t)\right)\right)\right| \leq \sum_{j=1}^{n} 2\left|\gamma_{i j}\right| \mathbb{M}_{j} \\
\left|\bigvee_{j=1}^{n} \delta_{i j} F_{j}\left(\dot{z}_{j}\left(t-\tau_{j}(t)\right)\right)\right| \leq \sum_{j=1}^{n}\left|\delta_{i j}\right|\left|F_{j}\left(\dot{z}_{j}\left(t-\tau_{j}(t)\right)\right)\right| \leq \sum_{j=1}^{n} 2\left|\delta_{i j}\right| \mathbb{M}_{j} .
\end{aligned}
$$

Then it leads to

$$
\begin{aligned}
\frac{d V(t)}{d t} \leq & \sum_{i=1}^{n}\left[-\zeta_{i} z_{i}^{2}(t)+\frac{p_{i}^{2}(t)}{2}+\frac{z_{i}^{2}(t)}{2}-h_{i 1} z_{i}^{2}(t)-h_{i 2}\left|z_{i}(t)\right|^{\kappa+1}-h_{i 3}\left|z_{i}(t)\right|^{\mu+1}\right] \\
& +\sum_{i=1}^{n}\left[-\bar{a}_{i} p_{i}^{2}(t)+\left|\bar{b}_{i}\right|\left(\frac{p_{i}^{2}(t)}{2}+\frac{z_{i}^{2}(t)}{2}\right)\right. \\
& +\sum_{j=1}^{n} \frac{\left|c_{j i}\right|}{2} \ell_{i} z_{i}^{2}(t)+\sum_{j=1}^{n} \frac{\left|c_{i j}\right|}{2} \ell_{j} p_{i}^{2}(t)-m_{i 1} p_{i}^{2}(t)-m_{i 2}\left|p_{i}(t)\right|^{\kappa+1} \\
& \left.+\left(\sum_{j=1}^{n} 2 \mathbb{M}_{j}\left(\left|d_{i j}\right|+\left|\eta_{i j}\right|+\left|e_{i j}\right|+\left|\alpha_{i j}\right|+\left|\beta_{i j}\right|+\left|\gamma_{i j}\right|+\left|\delta_{i j}\right|\right)-\varpi\right)\left|p_{i}(t)\right|-m_{i 3}\left|p_{i}(t)\right|^{\mu+1}\right]
\end{aligned}
$$

By using (27), we deduce that

$$
\begin{aligned}
\frac{d V(t)}{d t} \leq & \sum_{i=1}^{n}\left[-\zeta_{i}+\frac{1}{2}-h_{i 1}+\frac{\left|\bar{b}_{i}\right|}{2}+\sum_{j=1}^{n} \frac{\left|c_{j i}\right|}{2} \ell_{i}\right] z_{i}^{2}(t)-\sum_{i=1}^{n} h_{i 2}\left|z_{i}(t)\right|^{\kappa+1}-\sum_{i=1}^{n} h_{i 3}\left|z_{i}(t)\right|^{\mu+1} \\
& +\sum_{i=1}^{n}\left[\frac{1}{2}-\bar{a}_{i}+\frac{\left|\bar{b}_{i}\right|}{2}+\sum_{j=1}^{n} \frac{\left|c_{i j}\right|}{2} \ell_{j}-m_{i 1}\right] p_{i}^{2}(t)-\sum_{i=1}^{n} m_{i 2}\left|p_{i}(t)\right|^{\kappa+1}-\sum_{i=1}^{n} m_{i 3}\left|p_{i}(t)\right|^{\mu+1}
\end{aligned}
$$

At this stage, we notice that the delays have been removed from the calculus. So, the proof can be continued as for a delay-free system. We have

$$
\begin{aligned}
\frac{d V(t)}{d t} \leq & -\min \left\{\min _{1 \leq i \leq n}\left\{\rho_{i 1}\right\}, \min _{1 \leq i \leq n}\left\{\rho_{i 2}\right\}\right\}\left(\sum_{i=1}^{n} z_{i}^{2}(t)+\sum_{i=1}^{n} p_{i}^{2}(t)\right) \\
\leq & -\min \left\{\min _{1 \leq i \leq n}\left\{h_{i 2}\right\}, \min _{1 \leq i \leq n}\left\{m_{i 2}\right\}\right\}\left(\sum_{i=1}^{n}\left|z_{i}(t)\right|^{\kappa+1}+\sum_{i=1}^{n}\left|p_{i}(t)\right|^{\kappa+1}\right) \\
& -\min \left\{\min _{1 \leq i \leq n}\left\{h_{i 2}\right\}, \min _{1 \leq i \leq n}\left\{m_{i 2}\right\}\right\}\left(\sum_{i=1}^{n}\left|z_{i}(t)\right|^{\mu+1}+\sum_{i=1}^{n}\left|p_{i}(t)\right|^{\mu+1}\right) \\
\leq & -2 \min \left\{\min _{1 \leq i \leq n}\left\{\rho_{i 1}\right\}, \min _{1 \leq i \leq n}\left\{\rho_{i 2}\right\}\right\} V(t)
\end{aligned}
$$




$$
\begin{aligned}
& -\min \left\{\min _{1 \leq i \leq n}\left\{h_{i 2}\right\}, \min _{1 \leq i \leq n}\left\{m_{i 2}\right\}\right\} 2^{\frac{\kappa+1}{2}} V^{\frac{\kappa+1}{2}}(t) \\
& -\min \left\{\min _{1 \leq i \leq n}\left\{h_{i 3}\right\}, \min _{1 \leq i \leq n}\left\{m_{i 3}\right\}\right\} 2 n^{\frac{1-\mu}{2}} V^{\frac{\mu+1}{2}}(t) \\
\leq & -\underline{a} V(t)-\underline{b} V^{\frac{\kappa+1}{2}}(t)-\underline{c} V^{\frac{\mu+1}{2}}(t) .
\end{aligned}
$$

According to Lemma 4 the system (5) is fixed-time stable and the settling-time satisfies (11).

Remark 4.3. The delay-dependent controller (7) with fuzzy terms relies not only on the current state but also on its past state and its derivative. When the past state and its derivative cannot be measured, we must design the delay-free controller [28.).

Remark 4.4. The controller considered in Theorem 2 is a discontinuous one, while in some cases continuity is necessary. Under these circumstances, we can approximate the term $\operatorname{sign}\left(p_{i}(t)\right)$ by $\frac{p_{i}(t)}{p_{i}(t)+\xi}$, where $\xi$ is a small positive constant.

Corollary 3. Assume that $\left(\mathbf{A}_{\mathbf{1}}\right)-\left(\mathbf{A}_{\mathbf{3}}\right)$ hold and suppose that there exists $\theta>1$ such that $\kappa=1-\frac{1}{\theta}$ and $\mu=1+\frac{1}{\theta}$. If the conditions (8)-(10) and (27) are satisfied, then the system (5) is fixed-time stabilized under the feedback control law (7). Moreover, the settling-time $\mathbf{T}$ satisfies

$$
\mathbf{T} \leq \mathbf{T}_{8}^{\max }=\frac{\theta \pi}{\sqrt{\underline{b} \underline{c}}} .
$$

Proof. We have

$$
\begin{aligned}
\frac{d V(t)}{d t} & \leq-\underline{a} V(t)-\underline{b} V^{\frac{\kappa+1}{2}}(t)-\underline{c} V^{\frac{\mu+1}{2}}(t) \\
& \leq-\underline{b} V^{\frac{\kappa+1}{2}}(t)-\underline{c} V^{\frac{\mu+1}{2}}(t) .
\end{aligned}
$$

Since $\kappa=1-\frac{1}{\theta}, \mu=1+\frac{1}{\theta}$, we have $\frac{\kappa+1}{2}=1-\frac{1}{2 \theta}$ and $\frac{\mu+1}{2}=1-\frac{1}{2 \mu}$. It follows that

$$
\frac{d V(t)}{d t} \leq-\underline{b} V^{1-\frac{1}{2 \theta}}(t)-\underline{c} V^{1+\frac{1}{2 \theta}}(t) .
$$

From Lemma 3 , we deduce that the system (5) is fixed-time stable and the settling-time satisfies

$$
\mathbf{T} \leq \mathbf{T}_{8}^{\max }=\frac{\theta \pi}{\sqrt{\underline{b} \underline{c}}} .
$$

Remark 4.5. In Theorem 2, $\kappa$ and $\mu$ are only required to satisfy $0<\kappa<1$ and $\mu>1$, while in Corollary 3 . $\kappa$ and $\mu$ must be taken as $\kappa=1-\frac{1}{\theta}$ and $\mu=1+\frac{1}{\theta}$ with $\theta>1$. Therefore, Theorem 2 has some advantages over Corollary 3 for practical applications.

\section{Numerical simulations}

In this section, we present two numerical examples to illustrate the feasibility and the effectiveness of our main results. 
Example 1. Consider the following FNTINNs with time-varying delay:

$$
\begin{aligned}
\frac{d^{2} y_{i}(t)}{d t^{2}}= & -a_{i} \frac{d y_{i}(t)}{d t}-b_{i} y_{i}(t)+\sum_{j=1}^{n} c_{i j} f_{j}\left(y_{j}(t)\right)+\sum_{j=1}^{n} d_{i j} f_{j}\left(y_{j}\left(t-\tau_{j}(t)\right)\right) \\
& +\sum_{j=1}^{n} \eta_{i j} \int_{-\infty}^{t} K(t-s) f_{j}\left(y_{j}(s)\right) d s+\sum_{j=1}^{n} e_{i j} f_{j}\left(\dot{y}_{j}\left(t-\tau_{j}(t)\right)\right) \\
& +\sum_{j=1}^{n} g_{i j}(t) v_{j}(t)+\bigwedge_{j=1}^{n} T_{i j}(t) v_{j}(t)+\bigwedge_{j=1}^{n} \alpha_{i j} f_{j}\left(y_{j}\left(t-\tau_{j}(t)\right)\right) \\
& +\bigvee_{j=1}^{n} \beta_{i j} f_{j}\left(y_{j}\left(t-\tau_{j}(t)\right)\right)+\bigwedge_{j=1}^{n} \gamma_{i j} f_{j}\left(\dot{y}_{j}\left(t-\tau_{j}(t)\right)\right)+\bigvee_{j=1}^{n} \delta_{i j} f_{j}\left(\dot{y}_{j}\left(t-\tau_{j}(t)\right)\right) \\
& +\sum_{j=1}^{n} g_{i j}(t) v_{j}(t)+\bigvee_{j=1}^{n} S_{i j}(t) v_{j}(t)+\bigwedge_{j=1}^{n} T_{i j}(t) v_{j}(t)+I_{i}(t),
\end{aligned}
$$

where the parameters of the system are given as follows: $n=2$, for $i, j=1,2, a_{i}=5 ; b_{i}=7, g_{i j}(t)=$ $S_{i j}(t)=T_{i j}(t)=v_{j}(t)=1, \tau_{j}()=.\frac{|\cos (.)|}{2}, I_{1}(t)=I_{2}(t)=-4, f_{j}(x)=\frac{1}{2}(x+\sin (x))$ and $K_{j}(s)=\exp (-s)$. For $\zeta_{i}=0.3$, letting $p_{i}(t)=\frac{d z_{i}(t)}{d t}+\zeta_{i} z_{i}(t)$ then the system 31 can be expressed by adding the control variables as

$$
\left\{\begin{aligned}
\frac{d z_{i}(t)}{d t}= & -0.3 z_{i}(t)+p_{i}(t)+\hat{Q}_{i}(t) \\
\frac{d p_{i}(t)}{d t}= & -4.7 p_{i}(t)-5.59 z_{i}(t)+\sum_{j=1}^{2} c_{i j} f_{j}\left(z_{j}(t)\right)+\sum_{j=1}^{2} d_{i j} f_{j}\left(z_{j}\left(t-\tau_{j}(t)\right)\right) \\
& +\sum_{j=1}^{2} \eta_{i j} \int_{-\infty}^{t} K(t-s) f_{j}\left(z_{j}(s)\right) d s+\sum_{j=1}^{2} e_{i j} f_{j}\left(\dot{z}_{j}\left(t-\tau_{j}(t)\right)\right) \\
& +\bigwedge_{j=1}^{2} \alpha_{i j} f_{j}\left(z_{j}\left(t-\tau_{j}(t)\right)\right)+\bigvee_{j=1}^{2} \beta_{i j} f_{j}\left(z_{j}\left(t-\tau_{j}(t)\right)\right) \\
& +\bigwedge_{j=1}^{2} \delta_{i j} f_{j}\left(\dot{z}_{j}\left(t-\tau_{j}(t)\right)\right)+\bigvee_{j=1} \delta_{i j} f_{j}\left(\dot{z}_{j}\left(t-\tau_{j}(t)\right)\right)+\check{Q}_{i}(t)
\end{aligned}\right.
$$

with

$$
\begin{array}{ll}
C=\left(c_{i j}\right)=\left(\begin{array}{cc}
-1.9 & -2 \\
-0.9 & -3
\end{array}\right) \quad, \quad D=\left(d_{i j}\right)=\left(\begin{array}{cc}
1.5 & -0.5 \\
0.6 & 1.9
\end{array}\right), \\
\eta=\left(\eta_{i j}\right)=\left(\begin{array}{cc}
2 & -2 \\
0.9 & -3
\end{array}\right) \quad, \quad E=\left(e_{i j}\right)=\left(\begin{array}{cc}
-1.5 & 0.6 \\
-0.6 & 2
\end{array}\right), \\
\alpha=\left(\alpha_{i j}\right)=\left(\begin{array}{cc}
1.9 & -0.3 \\
-0.9 & 0.3
\end{array}\right) \quad, \quad \beta=\left(\beta_{i j}\right)=\left(\begin{array}{cc}
1.2 & -0.6 \\
0.5 & -0.8
\end{array}\right), \\
\gamma=\left(\gamma_{i j}\right)=\left(\begin{array}{cc}
1.7 & 0.3 \\
-1 & 0.4
\end{array}\right) \quad, \quad \delta=\left(\delta_{i j}\right)=\left(\begin{array}{cc}
1.5 & -0.2 \\
0.7 & -0.8
\end{array}\right) .
\end{array}
$$


In this case $f_{j}$ satisfies $\left(\mathbf{A}_{\mathbf{1}}\right)$ with $\ell_{j}=1$. Now, we choose the parameters of the controller (7) as follows

$$
\begin{aligned}
h_{11} & =6.78>-\zeta_{1}+\frac{1}{2}+\frac{\left|\bar{b}_{1}\right|}{2}+\sum_{j=1}^{2} \frac{\left|c_{j 1}\right|}{2} \ell_{1}=4.53 \\
h_{21} & =6>-\zeta_{2}+\frac{1}{2}+\frac{\left|\bar{b}_{2}\right|}{2}+\sum_{j=1}^{2} \frac{\left|c_{j 2}\right|}{2} \ell_{2}=3.65 \\
m_{11} & =3.5>-\bar{a}_{1}+\frac{1}{2}+\frac{\left|\bar{b}_{1}\right|}{2}+\sum_{j=1}^{2} \frac{\left|c_{1 j}\right|}{2} \ell_{j}=0.5 \\
m_{21}= & 3.5>-\bar{a}_{2}+\frac{1}{2}+\frac{\left|\bar{b}_{2}\right|}{2}+\sum_{j=1}^{2} \frac{\left|c_{2 j}\right|}{2} \ell_{j}=0.5 \\
h_{12}= & h_{22}=m_{12}=m_{22}=1.9>0 \\
h_{13}= & h_{23}=m_{13}=m_{23}=2.639>0
\end{aligned}
$$

and $0<\kappa=0.7<1 ; \quad \mu=1.3>1$. Finally, all the conditions of Theorem 1 are satisfied, and hence, the system $\sqrt{32}$ is fixed-time stabilized and the settling-time is given by $\mathbf{T}_{5}^{\max }=2.3596$. Taking $z_{1}(0)=-0.3, p_{1}(0)=0.4, z_{2}(0)=0.3, p_{2}(0)=-0.4$, the state trajectories of the system $(32)$ under the controller (7) are depicted in Figure 1.

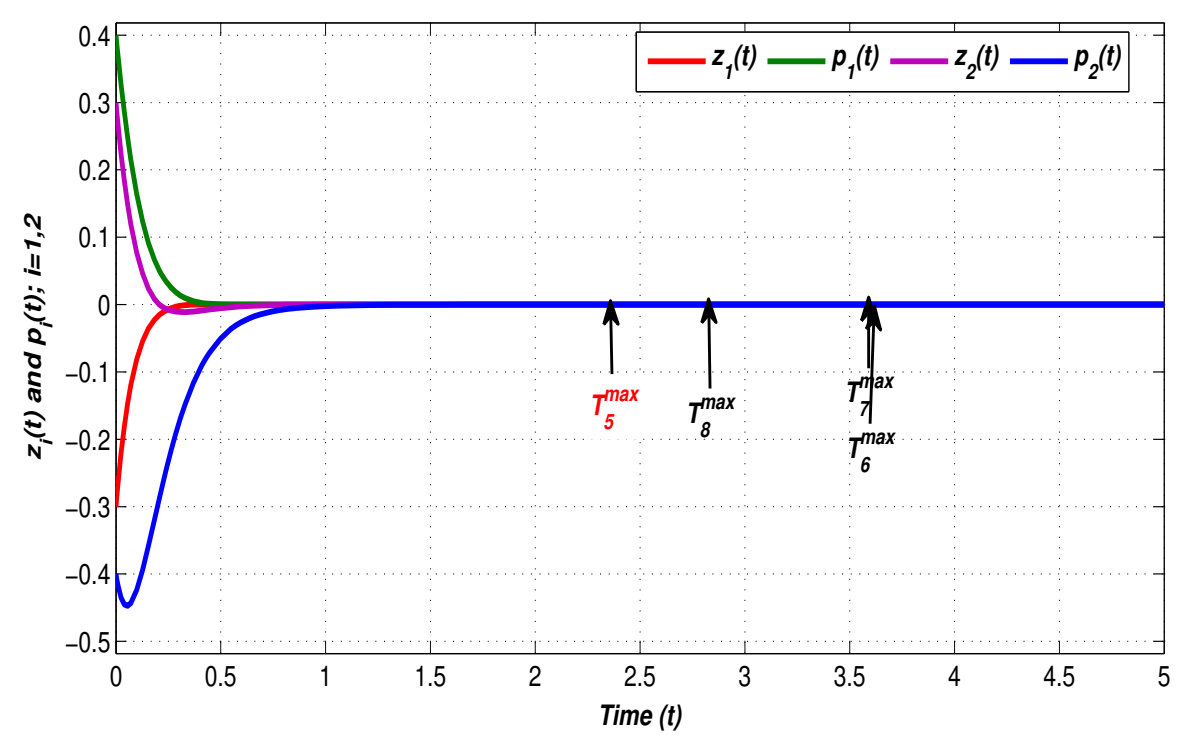

Figure 1: State trajectories of the system 32 under the controller 7 
Example 2. Consider the following FNTINNs with time-varying delay:

$$
\begin{aligned}
\frac{d^{2} y_{i}(t)}{d t^{2}}= & -a_{i} \frac{d y_{i}(t)}{d t}-b_{i} y_{i}(t)+\sum_{j=1}^{n} c_{i j} f_{j}\left(y_{j}(t)\right)+\sum_{j=1}^{n} d_{i j} f_{j}\left(y_{j}\left(t-\tau_{j}(t)\right)\right) \\
& +\sum_{j=1}^{n} \eta_{i j} \int_{-\infty}^{t} K(t-s) f_{j}\left(y_{j}(s)\right) d s+\sum_{j=1}^{n} e_{i j} f_{j}\left(\dot{y}_{j}\left(t-\tau_{j}(t)\right)\right) \\
& +\sum_{j=1}^{n} g_{i j}(t) v_{j}(t)+\bigwedge_{j=1}^{n} T_{i j}(t) v_{j}(t)+\bigwedge_{j=1}^{n} \alpha_{i j} f_{j}\left(y_{j}\left(t-\tau_{j}(t)\right)\right) \\
& +\bigvee_{j=1}^{n} \beta_{i j} f_{j}\left(y_{j}\left(t-\tau_{j}(t)\right)\right)+\bigwedge_{j=1}^{n} \gamma_{i j} f_{j}\left(\dot{y}_{j}\left(t-\tau_{j}(t)\right)\right)+\bigvee_{j=1}^{n} \delta_{i j} f_{j}\left(\dot{y}_{j}\left(t-\tau_{j}(t)\right)\right) \\
& +\sum_{j=1}^{n} g_{i j}(t) v_{j}(t)+\bigvee_{j=1}^{n} S_{i j}(t) v_{j}(t)+\bigwedge_{j=1}^{n} T_{i j}(t) v_{j}(t)+I_{i}(t),
\end{aligned}
$$

where the parameters of the system are given as follows: $n=2$, for $i, j=1,2, a_{i}=2 ; b_{i}=0.5, g_{i j}(t)=$ $S_{i j}(t)=T_{i j}(t)=v_{j}(t)=1, \tau_{j}()=1,. I_{1}(t)=I_{2}(t)=-4, f_{j}()=.\tanh (),. K(s)=\exp (-s)$,

$$
\begin{array}{cc}
C=\left(c_{i j}\right)=\left(\begin{array}{cc}
0.5 & 0.5 \\
-0.2 & -0.25
\end{array}\right) \quad, \quad D=\left(d_{i j}\right)=\left(\begin{array}{cc}
-0.15 & 0.13 \\
-0.3 & -0.1
\end{array}\right), \\
E=\left(e_{i j}\right)=\left(\begin{array}{cc}
-0.2 & 0.25 \\
-0.2 & -0.1
\end{array}\right) \quad, \quad \eta=\left(\eta_{i j}\right)=\left(\begin{array}{cc}
-0.15 & 0.13 \\
-0.3 & -0.1
\end{array}\right), \\
\left(\alpha_{i j}\right)=\left(\beta_{i j}\right)=\left(\begin{array}{ll}
-0.6 & 0.5 \\
-0.4 & 0.8
\end{array}\right) \quad, \quad\left(\gamma_{i j}\right)=\left(\delta_{i j}\right)=\left(\begin{array}{cc}
-0.6 & 0.5 \\
-0.4 & 0.8
\end{array}\right) .
\end{array}
$$

In this case $f_{j}$ satisfies $\left(\mathbf{A}_{\mathbf{1}}\right)$ and $\left(\mathbf{A}_{\mathbf{3}}\right)$ with $\ell_{j}=\mathbb{M}_{j}=1$ and $\int_{0}^{\infty} K(s) d s=1$. Now, we choose $\zeta_{1}=\zeta_{2}=1$ and the parameters of the controller (7) as follows

$$
\begin{aligned}
& h_{11}=0.5>-\zeta_{1}+\frac{1}{2}+\frac{\left|\bar{b}_{1}\right|}{2}+\sum_{j=1}^{2} \frac{\left|c_{j 1}\right|}{2} \ell_{1}=0.1 ; \\
& h_{21}=0.5>-\zeta_{2}+\frac{1}{2}+\frac{\left|\bar{b}_{2}\right|}{2}+\sum_{j=1}^{2} \frac{\left|c_{j 2}\right|}{2} \ell_{2}=0.125 ; \\
& m_{11}=3.5>-\bar{a}_{1}+\frac{1}{2}+\frac{\left|\bar{b}_{1}\right|}{2}+\sum_{j=1}^{2} \frac{\left|c_{1 j}\right|}{2} \ell_{j}=0.25 ; \\
& m_{21}= 3.5>-\bar{a}_{2}+\frac{1}{2}+\frac{\left|\bar{b}_{2}\right|}{2}+\sum_{j=1}^{2} \frac{\left|c_{2 j}\right|}{2} \ell_{j}=-0.025 ; \\
& h_{12}=h_{22}=m_{12}=m_{22}=2.0169>0 ; \\
& h_{13}=h_{23}=m_{13}=m_{23}=1.9503>0 ; \\
& \varpi=23>2 \sum_{j=1}^{2} \mathbb{M}_{j}\left(\left|d_{i j}\right|+\left|\eta_{i j}\right|+\left|e_{i j}\right|+\left|\alpha_{i j}\right|+\left|\beta_{i j}\right|+\left|\gamma_{i j}\right|+\left|\delta_{i j}\right|\right)=22.66
\end{aligned}
$$

and $0<\kappa=0.5<1 ; \mu=1.5>1$. Finally, all the conditions of Theorem 2 are satisfied, and hence, the system (33) is fixed-time stabilized and the settling-time is given by $\mathbf{T}_{5}^{\max }=2.0033$. Taking $z_{1}(0)=0.3, p_{1}(0)=-0.1, z_{2}(0)=-0.3, p_{2}(0)=0.1$, the state trajectories of the system 33 under the controller 28] are depicted in Figure 2. 


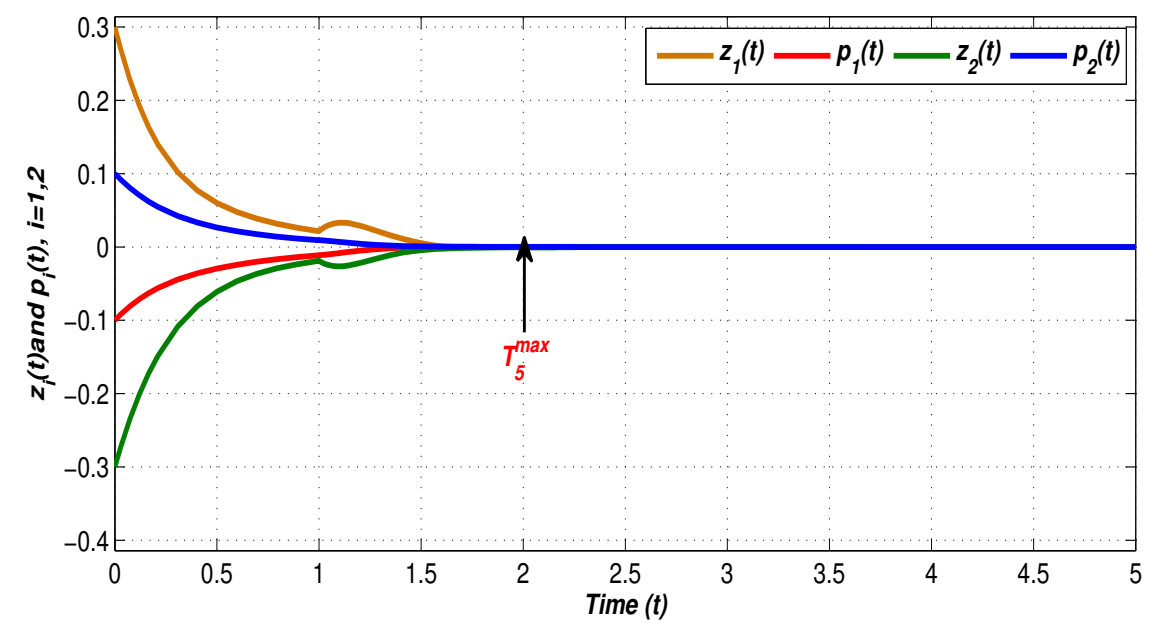

Figure 2: The state trajectories of the system 33 under the controller 28

Example 3. Consider the following FNTINNs with time-varying delay:

$$
\begin{aligned}
\frac{d^{2} y_{i}(t)}{d t^{2}}= & -a_{i} \frac{d y_{i}(t)}{d t}-b_{i} y_{i}(t)+\sum_{j=1}^{2} c_{i j} F_{j}\left(y_{j}(t)\right)+\sum_{j=1}^{2} d_{i j} F_{j}\left(y_{j}\left(t-\tau_{j}(t)\right)\right) \\
& +\sum_{j=1}^{2} g_{i j} v_{j}+\bigwedge_{j=1}^{2} T_{i j} v_{j}+\bigwedge_{j=1}^{2} \alpha_{i j} F_{j}\left(y_{j}\left(t-\tau_{j}(t)\right)\right) \\
& +\bigvee_{j=1}^{2} \beta_{i j} F_{j}\left(y_{j}\left(t-\tau_{j}(t)\right)\right)+\bigvee_{j=1}^{2} S_{i j} v_{j}+I_{i}
\end{aligned}
$$

where the parameters of system are given as follows: $n=2$, for $i, j=1,2, a_{i}=2.5, b_{i}=5, \tau_{j}()=.\frac{\exp (.)}{\exp (.)+1}$, $I_{i}=-\sum_{j=1}^{2} g_{i j} v_{j}-\bigwedge_{j=1}^{2} T_{i j} v_{j}-\bigvee_{j=1}^{2} S_{i j} v_{j}, F_{j}()=.\tanh ($.$) . In this case F_{j}$ satisfies $\left(\mathbf{A}_{\mathbf{1}}\right)$ and $\left(\mathbf{A}_{\mathbf{3}}\right)$ with $\ell_{j}=\mathbb{M}_{j}=1$. For $\zeta_{i}=0.5$, letting $p_{i}(t)=\frac{d z_{i}(t)}{d t}+\zeta_{i} z_{i}(t)$ then the system 34 can be expressed by adding the control variables as

$$
\left\{\begin{aligned}
\frac{d z_{i}(t)}{d t}= & -0.5 z_{i}(t)+p_{i}(t)+\hat{Q}_{i}(t) \\
\frac{d p_{i}(t)}{d t}= & -2 p_{i}(t)-4 z_{i}(t)+\sum_{j=1}^{2} c_{i j} F_{j}\left(z_{j}(t)\right) \\
& +\sum_{j=1}^{2} d_{i j} F_{j}\left(z_{j}\left(t-\tau_{j}(t)\right)\right)+\bigwedge_{j=1}^{2} \alpha_{i j} F_{j}\left(z_{j}\left(t-\tau_{j}(t)\right)\right) \\
& +\bigvee_{j=1}^{2} \beta_{i j} F_{j}\left(z_{j}\left(t-\tau_{j}(t)\right)\right)+\check{Q}_{i}(t)
\end{aligned}\right.
$$

with

$$
\begin{aligned}
& C=\left(c_{i j}\right)=\left(\begin{array}{cc}
-0.2 & -0.15 \\
-0.1 & -0.4
\end{array}\right) \quad, \quad D=\left(d_{i j}\right)=\left(\begin{array}{cc}
-0.6 & -0.6 \\
0.1 & -0.03
\end{array}\right), \\
& \alpha=\left(\alpha_{i j}\right)=\left(\begin{array}{ll}
-0.8 & -0.1 \\
-0.2 & -0.1
\end{array}\right) \quad, \quad \beta=\left(\beta_{i j}\right)=\left(\begin{array}{cc}
-0.5 & -0.5 \\
0.6 & -0.2
\end{array}\right) .
\end{aligned}
$$


Now, choosing the parameters of the controller 28 as follows

$$
\begin{aligned}
h_{11} & =5>-\zeta_{1}+\frac{1}{2}+\frac{\left|\bar{b}_{1}\right|}{2}+\sum_{j=1}^{2} \frac{\left|c_{j 1}\right|}{2} \ell_{1}=2.15 ; \\
h_{21} & =5>-\zeta_{2}+\frac{1}{2}+\frac{\left|\bar{b}_{2}\right|}{2}+\sum_{j=1}^{2} \frac{\left|c_{j 2}\right|}{2} \ell_{2}=2.275 ; \\
m_{11} & =5 \geq-\bar{a}_{1}+\frac{1}{2}+\frac{\left|\bar{b}_{1}\right|}{2}+\sum_{j=1}^{2} \frac{\left|c_{1 j}\right|}{2} \ell_{j}=0.8 ; \\
m_{21} & =5 \geq-\bar{a}_{2}+\frac{1}{2}+\frac{\left|\bar{b}_{2}\right|}{2}+\sum_{j=1}^{2} \frac{\left|c_{2 j}\right|}{2} \ell_{j}=0.75 ; \\
h_{12} & =h_{22}=m_{12}=m_{22}=2.0169>0 ; \\
h_{13} & =h_{23}=m_{13}=m_{23}=1.9503>0 ; \\
\varpi & =19.46 ; \quad 0<\kappa=0.5<1 ; \quad \mu=1.5>1 ;
\end{aligned}
$$

all the conditions of Theorem 2 are verified, and hence, the system (35) is fixed-time stabilized and the settling-time is given by $\mathbf{T}_{5}^{\max }=1.3449$. Taking $z_{1}(0)=-0.5, p_{1}(0)=-0.7, z_{2}(0)=0.9, p_{2}(0)=0.5$, the state trajectories of the system (35) under the controller (28) are depicted in Figure 3 .

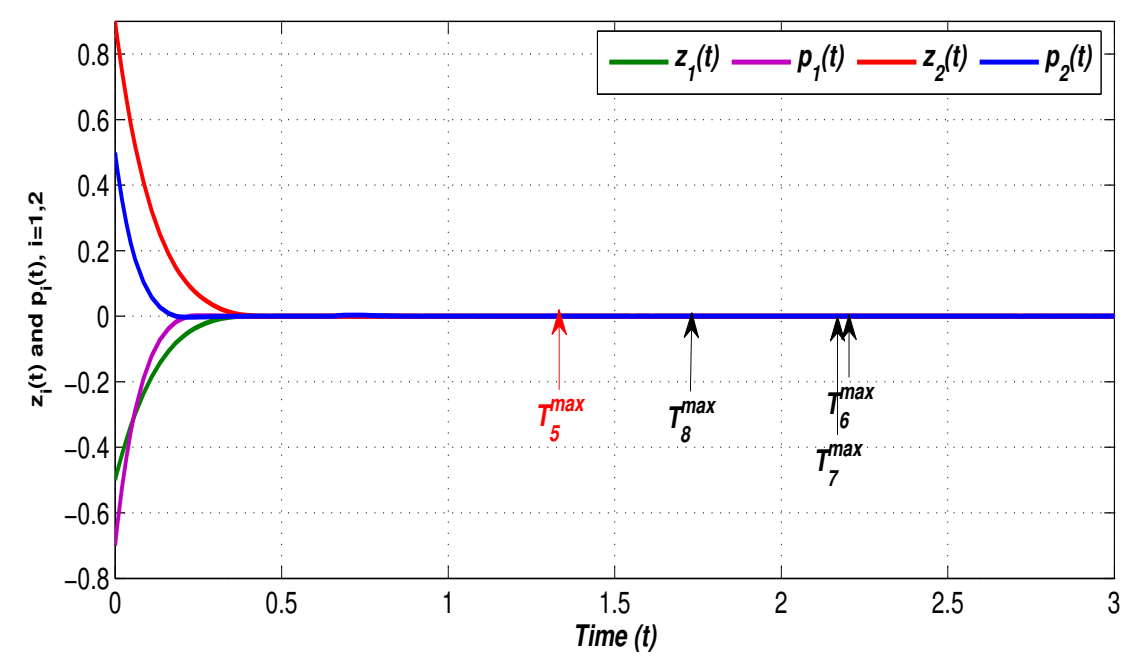

Figure 3: The state trajectories of the system 35 under the controller 28

Remark 5.1. Every upper bound estimate of the settling-time is obtained by Lyapunov-based theoretical analysis. Due to the conservativeness of some inequalities involved in this technique, the upper bound estimates of the settling-time obtained with the theoretical results may be much larger than the real settling-time as illustrated in the numerical simulations, see Figures 1 and 3 . As shown in Examples 1 and 3 with the same controller parameters, $\mathbf{T}_{5}^{\max }$ is shorter than $\mathbf{T}_{6}^{\max }, \mathbf{T}_{7}^{\max }$, and $\mathbf{T}_{8}^{\max }$. This is summed up in Table 1 . 


\begin{tabular}{|l|c|c|c|c|}
\hline Settling-time & $\mathbf{T}_{5}^{\max }$ & $\mathbf{T}_{6}^{\max }$ & $\mathbf{T}_{7}^{\max }$ & $\mathbf{T}_{8}^{\max }$ \\
\hline Example 1 & 2.3596 & 3.6133 & 3.6024 & 2.8293 \\
\hline Example 3 & 1.3449 & 2.2047 & 2.1994 & 1.7274 \\
\hline
\end{tabular}

Table 1: Comparisons between $\mathbf{T}_{5}^{\max }, \mathbf{T}_{6}^{\max }, \mathbf{T}_{7}^{\max }$, and $\mathbf{T}_{8}^{\max }$.

Remark 5.2. In both Examples 1 and 3 , if we choose some larger values for $h_{11}, h_{21}, m_{11}, m_{21}$ and keep the other controller parameters unchanged, then the obtained $\mathbf{T}_{5}^{\max }$ can be shorter but $\mathbf{T}_{6}^{\max }, \mathbf{T}_{7}^{\max }$ and $\mathbf{T}_{8}^{\max }$ remains unchanged.

Remark 5.3. The fuzzy neural networks are investigated in [7, 29, 47]. These models are first order differential equations without neutral terms and distributed time-varying delays. While, in this paper, a class of fuzzy neutral-type inertial neural networks is studied with mixed time-varying delay. Furthermore, numerical examples show that the new fixed-time stability theorem considered in this paper can provide a more accurate upper settling-time estimation for NNs compared to previous studies.

Remark 5.4. In [13], some fixed-time synchronization results about inertial memristor based NNs with discrete delay are obtained. Compared with [13], our results provide a lower upper settling-time estimation. In addition, the fuzzy term was not taken account in [13]. Finally, the time delays in [13] are discrete, while they are time-varying in our article.

Remark 5.5. The stabilization of NNs has been developed to solve problems of classification, prediction, categorization, optimization, pattern recognition, and secure communication [12, 38, 39, 48]. In these application fields, sometimes it is necessary to complete certain tasks within a finite time or fixed time. Contrary to the finite-time stabilization, the settling-time obtained with fixed-time stabilization is independent on the initial conditions. In some areas, such as pattern recognition or secure communication, the initial values are usually hard to be acquired [40]. In this case, fixed-time stabilization is more accurate and applicable than finite-time stabilization.

\section{Conclusion}

The problem of fixed-time stabilization for a class of fuzzy neutral-type inertial neural networks (FNTINNs) was investigated. By using the new fixed-time stability theorems for dynamical systems, two different feedback control laws were designed to ensure the fixed-time stabilization of FNTINNs, which include and extend some earlier works. On the one hand, our results extend the results given in [12, 44, 45, 46] where fuzzy inertial terms are not taken into account. On the other hand, a tighter upper bound estimate of the settlingtime is derived. Finally, the effectiveness of our proposed approach has been illustrated in simulation with three examples. In recent decades, stochastic differential equations have been widely considered by many researchers since they can be applied to various fields such as science and engineering. It is known that one important problem of stochastic differential equations is the stability analysis [43, 49, 50, 51, In future works, we would like to extend our results to stochastic neural networks and more general neutral-type of delayed stochastic inertial neural networks, such as high-order neutral-type of delayed stochastic inertial neural networks models, high-order Hopfield bidirectional associative memory neural networks [52, Clifford inertial neural networks models 53,54 .

\section{References}

[1] T. Yang, L.-B. Yang, The global stability of fuzzy cellular neural network, IEEE Transactions on Circuits and Systems I: Fundamental Theory and Applications 43 (10) (1996) 880-883.

[2] T. Yang, L.-B. Yang, C. W. Wu, L. O. Chua, Fuzzy cellular neural networks: theory, in: 4th IEEE International Workshop on Cellular Neural Networks and Their Applications Proceedings (CNNA-96), IEEE, 1996, pp. 181-186.

[3] T. Yang, L.-B. Yang, Application of fuzzy cellular neural networks to Euclidean distance transformation, IEEE Transactions on Circuits and Systems I: Fundamental Theory and Applications 44 (3) (1997) 242-246. 
[4] T. Yang, L.-B. Yang, Fuzzy cellular neural network: A new paradigm for image processing, International Journal of Circuit Theory and Applications 25 (6) (1997) 469-481.

[5] F. Russo, G. Ramponi, Fuzzy operator for sharpening of noisy images, Electronics Letters 28 (18) (1992) 1715-1717.

[6] T. Yang, L.-B. Yang, C. W. Wu, L. O. Chua, Fuzzy cellular neural networks: applications, in: 1996 Fourth IEEE International Workshop on Cellular Neural Networks and Their Applications Proceedings (CNNA-96), IEEE, 1996, pp. $225-230$.

[7] Q. Zhu, X. Li, Exponential and almost sure exponential stability of stochastic fuzzy delayed Cohen-Grossberg neural networks, Fuzzy Sets and Systems 203 (2012) 74-94.

[8] J. Chen, J. H. Park, S. Xu, Stability analysis for neural networks with time-varying delay via improved techniques, IEEE transactions on cybernetics 49 (12) (2018) 4495-4500.

[9] T. H. Lee, H. M. Trinh, J. H. Park, Stability analysis of neural networks with time-varying delay by constructing novel Lyapunov functionals, IEEE Transactions on Neural Networks and Learning Systems 29 (9) (2017) $4238-4247$.

[10] W. Wang, Finite-time synchronization for a class of fuzzy cellular neural networks with time-varying coefficients and proportional delays, Fuzzy Sets and Systems 338 (2018) 40-49.

[11] D. W. Wheeler, W. Schieve, Stability and chaos in an inertial two-neuron system, Physica D: Nonlinear Phenomena 105 (4) (1997) 267-284.

[12] A. M. Alimi, C. Aouiti, E. A. Assali, Finite-time and fixed-time synchronization of a class of inertial neural networks with multi-proportional delays and its application to secure communication, Neurocomputing 332 (2019) $29-43$.

[13] C. Chen, L. Li, H. Peng, Y. Yang, Fixed-time synchronization of inertial memristor-based neural networks with discrete delay, Neural Networks 109 (2019) 81-89.

[14] S. Nazrulla, H. K. Khalil, Robust stabilization of non-minimum phase nonlinear systems using extended high-gain observers, IEEE Transactions on Automatic Control 56 (4) (2010) 802-813.

[15] H. Sang, J. Zhao, Exponential synchronization and $l_{-2}$-gain analysis of delayed chaotic neural networks via intermittent control with actuator saturation, IEEE Transactions on Neural Networks and Learning Systems 30 (12) (2019) $3722-3734$.

[16] S. P. Bhat, D. S. Bernstein, Finite-time stability of continuous autonomous systems, SIAM Journal on Control and Optimization 38 (3) (2000) 751-766.

[17] E. Moulay, W. Perruquetti, Finite time stability and stabilization of a class of continuous systems, Journal of Mathematical analysis and applications 323 (2) (2006) 1430-1443.

[18] H. Du, S. Li, C. Qian, Finite-time attitude tracking control of spacecraft with application to attitude synchronization, IEEE Transactions on Automatic Control 56 (11) (2011) 2711-2717.

[19] B. Jiang, Q. Hu, M. I. Friswell, Fixed-time attitude control for rigid spacecraft with actuator saturation and faults, IEEE Transactions on Control Systems Technology 24 (5) (2016) 1892-1898.

[20] S.-K. Lee, D. Jang, Translation, rotation and scale invariant pattern recognition using spectral analysis and hybrid geneticneural-fuzzy networks, Computers \& Industrial Engineering 30 (3) (1996) 511-522.

[21] J. Savković-Stevanović, A neural network model for analysis and optimization of processes, Computers \& Chemical Engineering 17 (1993) S411-S416.

[22] J. Jian, L. Duan, Finite-time synchronization for fuzzy neutral-type inertial neural networks with time-varying coefficients and proportional delays, Fuzzy Sets and Systems 381 (2020) 51-67.

[23] J. Cao, R. Li, Fixed-time synchronization of delayed memristor-based recurrent neural networks, Science China Information Sciences 60 (3) (2017) 032201.

[24] A. Polyakov, Nonlinear feedback design for fixed-time stabilization of linear control systems, IEEE Transactions on Automatic Control 57 (8) (2012) 2106-2110.

[25] H. Li, C. Li, T. Huang, W. Zhang, Fixed-time stabilization of impulsive Cohen-Grossberg BAM neural networks, Neural Networks 98 (2018) 203-211.

[26] S. Lakshmanan, C. P. Lim, M. Prakash, S. Nahavandi, P. Balasubramaniam, Neutral-type of delayed inertial neural networks and their stability analysis using the LMI approach, Neurocomputing 230 (2017) 243-250.

[27] Z. Guo, S. Gong, T. Huang, Finite-time synchronization of inertial memristive neural networks with time delay via delay-dependent control, Neurocomputing 293 (2018) 100-107.

[28] N. Cui, H. Jiang, C. Hu, A. Abdurahman, Finite-time synchronization of inertial neural networks, Journal of the Association of Arab Universities for Basic and Applied Sciences 24 (2017) 300-309.

[29] C. Aouiti, F. Miaadi, A new fixed-time stabilization approach for neural networks with time-varying delays, Neural Computing and Applications (2019) 1-15

[30] H. Wang, Q. Zhu, Finite-time stabilization of high-order stochastic nonlinear systems in strict-feedback form, Automatica 54 (2015) 284-291.

[31] Z. Zuo, Q.-L. Han, B. Ning, Fixed-Time Cooperative Control of Multi-Agent Systems, Control Engineering, Springer, 2019.

[32] C. Chen, L. Li, H. Peng, Y. Yang, L. Mi, H. Zhao, A new fixed-time stability theorem and its application to the fixed-time synchronization of neural networks, Neural Networks 123 (2020) 412-419.

[33] R. Rakkiyappan, S. Premalatha, A. Chandrasekar, J. Cao, Stability and synchronization analysis of inertial memristive neural networks with time delays, Cognitive neurodynamics 10 (5) (2016) 437-451.

[34] S. Lu, Y. Gao, Exponential stability in lagrange sense for inertial neural networks with time-varying delays, Neurocomputing 333 (2019) 41-52.

[35] Z. Zhang, Z. Quan, Global exponential stability via inequality technique for inertial BAM neural networks with time delays, Neurocomputing 151 (2015) 1316-1326.

[36] K. Yuan, J. Cao, J. Deng, Exponential stability and periodic solutions of fuzzy cellular neural networks with time-varying 
delays, Neurocomputing 69 (13-15) (2006) 1619-1627.

[37] K. O. Friedrichs, Advanced ordinary differential equations, CRC Press, 1985.

[38] M. Forti, P. Nistri, M. Quincampoix, Generalized neural network for nonsmooth nonlinear programming problems, IEEE Transactions on Circuits and Systems I: Regular Papers 51 (9) (2004) 1741-1754.

[39] C. Huang, J. Cao, M. Xiao, A. Alsaedi, F. E. Alsaadi, Controlling bifurcation in a delayed fractional predator-prey system with incommensurate orders, Applied Mathematics and Computation 293 (2017) 293-310.

[40] Z. Zuo, Q.-L. Han, B. Ning, X. Ge, X.-M. Zhang, An overview of recent advances in fixed-time cooperative control of multiagent systems, IEEE Transactions on Industrial Informatics 14 (6) (2018) 2322-2334.

[41] W. Ding, M. Han, Synchronization of delayed fuzzy cellular neural networks based on adaptive control, Physics Letters A $372(26)(2008)$ 4674-4681.

[42] R. Jia, Finite-time stability of a class of fuzzy cellular neural networks with multi-proportional delays, Fuzzy Sets and Systems 319 (2017) 70-80.

[43] Q. Zhu, Stability analysis of stochastic delay differential equations with lévy noise, Systems \& Control Letters 118 (2018) $62-68$.

[44] Z. Zuo, Non-singular fixed-time terminal sliding mode control of non-linear systems, IET control theory \& applications 9 (4) (2014) 545-552.

[45] Z. Zuo, M. Defoort, B. Tian, Z. Ding, Fixed-time stabilization of second-order uncertain multivariable nonlinear systems, in: 2016 35th Chinese Control Conference (CCC), IEEE, 2016, pp. 907-912.

[46] M. Zheng, L. Li, H. Peng, J. Xiao, Y. Yang, Y. Zhang, H. Zhao, Globally fixed-time synchronization of coupled neutral-type neural network with mixed time-varying delays, PloS One 13 (1) (2018) e0191473.

[47] Y. Liu, Y. Sun, Fixed-Time Synchronization of Fuzzy Cellular Neural Networks With Time-Varying Delays and Discontinuous Activations, IEEE Access 8 (2020) 65801-65811.

[48] C. Aouiti, E. A. Assali, Stability analysis for a class of impulsive high-order Hopfield neural networks with leakage timevarying delays, Neural Computing and Applications 31 (11) (2019) 7781-7803.

[49] Q. Zhu, Stabilization of stochastic nonlinear delay systems with exogenous disturbances and the event-triggered feedback control, IEEE Transactions on Automatic Control 64 (9) (2019) 3764-3771.

[50] Q. Zhu, T. Huang, Stability analysis for a class of stochastic delay nonlinear systems driven by g-brownian motion, Systems \& Control Letters 140 (2020) 104699.

[51] W. Hu, Q. Zhu, H. R. Karimi, Some improved Razumikhin stability criteria for impulsive stochastic delay differential systems, IEEE Transactions on Automatic Control 64 (12) (2019) 5207-5213.

[52] C. Aouiti, R. Sakthivel, F. Touati, Global dissipativity of high-order Hopfield bidirectional associative memory neural networks with mixed delays, Neural Computing and Applications 32 (14) (2020) 10183-10197.

[53] C. Aouiti, I. Ben Gharbia, Dynamics behavior for second-order neutral Clifford differential equations: inertial neural networks with mixed delays, Computational and Applied Mathematics 39 (2020) 1-31.

[54] C. Aouiti, F. Dridi, Weighted pseudo almost automorphic solutions for neutral type fuzzy cellular neural networks with mixed delays and D operator in Clifford algebra, International Journal of Systems Science (2020) 1-23. 